\title{
Existence of solutions for integral boundary value problems of singular Hadamard-type fractional differential equations on infinite interval
}

Weiwei Liu', Lishan Liu' ${ }^{*}$ and Yonghong Wu²

"Correspondence:

mathlls@163.com

'School of Mathematics Sciences,

Qufu Normal University, Qufu,

People's Republic of China

Full list of author information is

available at the end of the article

\section{Springer}

\begin{abstract}
We consider the existence of solutions for the following Hadamard-type fractional differential equations:

$$
\left\{\begin{array}{l}
{ }^{H} D^{\alpha} u(t)+q(t) f\left(t, u(t),{ }^{H} D^{\beta_{1}} u(t),{ }^{H} D^{\beta_{2}} u(t)\right)=0, \quad 1<t<+\infty, \\
u(1)=0, \\
{ }^{H} D^{\alpha-2} u(1)=\int_{1}^{+\infty} g_{1}(s) u(s) \frac{d s}{s}, \\
{ }^{H} D^{\alpha-1} u(+\infty)=\int_{1}^{+\infty} g_{2}(s) u(s) \frac{d s}{s},
\end{array}\right.
$$

where $2<\alpha \leq 3,0<\beta_{1} \leq \alpha-2<\beta_{2} \leq \alpha-1, f: J \times \mathbb{R}^{3} \rightarrow \mathbb{R}$ satisfies the q-Carathéodory condition, $q_{1} g_{1}, g_{2}: J \rightarrow \mathbb{R}^{+}$are nonnegative, where $J=[1,+\infty)$. Nonlinear term $f$ is dependent on the fractional derivative of lower order $\beta_{1}, \beta_{2}$, which creates additional complexity to verify the existence of solutions. The singularity occurring in our problem is associated with ${ }^{H} D^{\beta_{2}} u \in C(1,+\infty)$ at the left endpoint $t=1$ (if $\beta_{2}<\alpha-1$ ).
\end{abstract}

MSC: 34B18; 34B10; 34B15

Keywords: Hadamard-type fractional differential equation; Carathéodory condition; Infinite interval; Fixed point theory

\section{Introduction}

Fractional differential equations arise from various applications including in a variety of fields of mathematical and natural science. Fractional boundary value problems can more accurately describe the nature of practical problems, they have solved a large number of applications in different kinds of fields such as viscoelasticity, biomedical engineering, mechanical, anomalous diffusion, etc. Therefore, they have become a research hot-spot. A variety of techniques, such as the method of mixed monotone operator, topological degree method, monotone iterative technique, etc., have been applied to obtain the existence of solutions for fractional boundary value problems (see [1-32]).

(c) The Author(s) 2020. This article is licensed under a Creative Commons Attribution 4.0 International License, which permits use, sharing, adaptation, distribution and reproduction in any medium or format, as long as you give appropriate credit to the original author(s) and the source, provide a link to the Creative Commons licence, and indicate if changes were made. The images or other third party material in this article are included in the article's Creative Commons licence, unless indicated otherwise in a credit line to the material. If material is not included in the article's Creative Commons licence and your intended use is not permitted by statutory regulation or exceeds the permitted use, you will need to obtain permission directly from the copyright holder. To view a copy of this licence, visit http://creativecommons.org/licenses/by/4.0/. 
Apart from the common differential equation with Riemann-Liouville and Caputo fractional derivative, there are also several kinds of fractional derivatives: Hadamard, ErdelyiKober, Hilfer, and so on. Here we emphasize that the studies about Hadamard fractional differential equations are still at the early stage and need further investigation. More details and recent contributions to the topic can be found in [33-51] and the references therein.

In [36], the author used the fixed point index to study the existence of positive solutions for a system of nonlinear Hadamard fractional differential equations involving coupled integral boundary conditions:

$$
\left\{\begin{array}{l}
D^{\beta} u(t)+f_{1}(t, u(t), v(t)), \quad t \in[1, e], \\
D^{\beta} v(t)+f_{2}(t, u(t), v(t)), \quad t \in[1, e], \\
u(1)=v(1)=u^{\prime}(1)=v^{\prime}(1)=0, \\
u(e)=\int_{1}^{e} h(s) v(s) \frac{d s}{s}, \\
v(e)=\int_{1}^{e} g(s) u(s) \frac{d s}{s},
\end{array}\right.
$$

where $\beta \in(2,3], f_{1}, f_{2}$ are nonnegative continuous functions on $[1, e] \times \mathbb{R}^{+} \times \mathbb{R}^{+}$.

Ardjouni in [45] employed the Schauder and Banach fixed point theorems and the method of upper and lower solutions to show the existence and uniqueness of a positive solution for nonlinear Hadamard fractional differential equations with integral boundary conditions:

$$
\left\{\begin{array}{l}
D_{1}^{\alpha} x(t)+f(t, x(t))=D_{1}^{\beta} g(t, x(t)), \quad t \in[1, e], \\
x(1)=0, \quad x(e)=\frac{1}{\Gamma(\alpha-\beta)} \int_{1}^{e}\left(\log \frac{e}{s}\right)^{\alpha-\beta-1} g(s, x(s)) \frac{d s}{s},
\end{array}\right.
$$

where $1<\alpha \leq 2,0<\beta \leq \alpha-1, g, f:[1, e] \times[0, \infty) \rightarrow[0, \infty)$ are given continuous functions, $g$ is nondecreasing on $x$, and $f$ does not require any monotone assumption.

In [46], Pei et al. investigated the following boundary value problem of Hadamard fractional integro-differential equations on infinite domain:

$$
\left\{\begin{array}{l}
{ }^{H} D^{\alpha} u(t)+f\left(t, u(t),{ }^{H} I^{\gamma} u(t),{ }^{H} D^{\alpha-1} u(t)\right)=0, \quad 1<\alpha<2, t \in(1, \infty), \\
u(1)=0, \quad{ }^{H} D^{\alpha-1} u(\infty)=\sum_{i=1}^{m} \lambda_{i}{ }^{H} I^{\beta_{i}} u(\eta)
\end{array}\right.
$$

where $\gamma, \beta_{i}, \lambda_{i} \geq 0(i=1,2, \ldots, m)$ are given constants and $\eta, \beta_{i}, \lambda_{i}$ satisfy $\Gamma(\alpha)>$ $\sum_{i=1}^{m} \frac{\lambda_{i} \Gamma(\alpha)}{\Gamma\left(\alpha+\beta_{i}\right)} \times(\log t)^{\alpha+\beta_{i}-1}$. The nonlinear term $f$ is nondecreasing with respect to the second, third, and last variables. By use of the monotone iterative method, the authors obtained not only the existence of positive solutions for Hadamard fractional integrodifferential equations on infinite intervals, but also the minimal and maximal positive solutions and two explicit monotone iterative sequences converging to the extremal solution.

El-Sayed and Gaafar [47] established the existence of positive solutions to the following singular nonlinear Hadamard-type fractional differential equations with infinite-point boundary conditions or integral boundary condition:

$$
\begin{aligned}
& { }_{H} D^{\gamma} v(t)+f\left(t, v(t),{ }_{H} D^{\delta} v(t), v^{\prime}(t)\right)=0, \quad \text { a.e. } t \in(1, e), \\
& v(1)=0, \quad v(e)=v_{0}+\lambda \int_{1}^{e} v(\Phi(\xi)) \frac{\Phi^{\prime}(\xi)}{\Phi(\xi)} d \xi
\end{aligned}
$$


or

$$
v(1)=0, \quad v(e)=v_{0}+\lambda \sum_{j=1}^{\infty} a_{j} v\left(\Phi\left(\eta_{j}\right)\right)
$$

where $1<\gamma<2,0<\delta<1,1 \leq \gamma-\delta<2, \lambda, v_{0}, a_{j}$ are nonnegative constants. $f:[1, e] \times \mathbb{R}^{+} \times$ $\mathbb{R}^{2}$ is an $L_{p}$ Carathéodory positive function.

In [2], Hao et al. considered a boundary value problem of fractional differential equation inclusions of Riemann-Liouville type on the infinite interval:

$$
\left\{\begin{array}{l}
D_{0+}^{\alpha} u(t)+f\left(t, u(t), D_{0+}^{\alpha-2} u(t), D_{0+}^{\alpha-1} u(t)\right)=0, \quad t \in(0, \infty) \\
u(0)=u^{\prime}(0)=0, \quad D^{\alpha-1} u(\infty)=\xi I_{0+}^{\beta} u(\eta)
\end{array}\right.
$$

where $2<\alpha \leq 3, \beta>0, \xi, \eta \geq 0, \Gamma(\alpha+\beta)>\xi \eta^{\alpha+\beta-1}, f \in C([0,+\infty] \times \mathbb{R} \times \mathbb{R} \times \mathbb{R}, \mathbb{R})$. Under suitable growth conditions of the nonlinear term $f$, by using the Schauder fixed point theorem and Banach contraction mapping principle, the authors showed the existence and uniqueness results of solutions.

Inspired by the works mentioned above, we will study the existence of solutions for the following boundary value problem for Hadamard fractional differential equations:

$$
{ }^{H} D^{\alpha} u(t)+q(t) f\left(t, u(t),{ }^{H} D^{\beta_{1}} u(t),{ }^{H} D^{\beta_{2}} u(t)\right)=0, \quad 1<t<+\infty,
$$

supplemented with Hadamard integral boundary conditions

$$
\left\{\begin{array}{l}
u(1)=0, \\
{ }^{H} D^{\alpha-2} u(1)=\int_{1}^{+\infty} g_{1}(s) u(s) \frac{d s}{s}, \\
{ }^{H} D^{\alpha-1} u(+\infty)=\int_{1}^{+\infty} g_{2}(s) u(s) \frac{d s}{s},
\end{array}\right.
$$

$2<\alpha \leq 3,0<\beta_{1} \leq \alpha-2<\beta_{2} \leq \alpha-1, q \in C\left(J, \mathbb{R}^{+}\right), J=[1,+\infty)$, and $0<\int_{1}^{+\infty} q(s) \frac{d s}{s}<+\infty$, $f: J \times \mathbb{R}^{3} \rightarrow \mathbb{R}$ satisfies the $q$-Carathéodory condition, $g_{1}, g_{2}: J \rightarrow \mathbb{R}^{+}$are nonnegative functions. ${ }^{H} D^{\alpha}$ represents a Hadamard fractional derivative of order $\alpha$.

We say that $f$ satisfies the $q$-Carathéodory condition on $J \times \mathbb{R}^{3} \rightarrow \mathbb{R}$, if

(1) for each $(u, v, w) \in \mathbb{R}^{3}$, the mapping $t \rightarrow f(t, u, v, w)$ is measurable on $J$;

(2) for a.e. $t \in J$, the mapping

$(u, v, w) \rightarrow f\left(t,\left(1+(\log t)^{\alpha-1}\right) u,\left(1+(\log t)^{\alpha-\beta_{1}-1}\right) v,\left(1+(\log t)^{\alpha-\beta_{2}}\right) \frac{w}{(\log t)^{2+\beta_{2}-\alpha}}\right)$ is continuous on $\mathbb{R}^{3}$;

(3) for each $r>0$, there exists a nonnegative function $f_{r}$ satisfying $\int_{1}^{\infty} q(s) f_{r}(s) \frac{d s}{s}<+\infty$ such that, for any $u, v, w \in \mathbb{R}$ with $\max \{u, v, w\} \leq r$,

$$
\left|f\left(t,\left(1+(\log t)^{\alpha-1}\right) u,\left(1+(\log t)^{\alpha-\beta_{1}-1}\right) v, \frac{1+(\log t)^{\alpha-\beta_{2}}}{(\log t)^{2+\beta_{2}-\alpha}} w\right)\right| \leq f_{r}(t), \quad \text { for a.e. } t \in J
$$

Compared with [2], in this paper, the nonlinear term $f$ contains lower Hadamard fractional derivatives ${ }^{H} D^{\beta_{1}},{ }^{H} D^{\beta_{2}}$, which are not only the particular case ${ }^{H} D^{\alpha-1},{ }^{H} D^{\alpha-2}$. Furthermore, when $\beta_{2}<\alpha-1, \lim _{t \rightarrow 1+}\left({ }^{H} D^{\beta_{2}} u\right)(t)=\infty$, the singularity creates additional complexity to verify the existence of solutions. 
This paper is organized as follows. Section 2 contains some important lemmas, which play a key role in the study, and presents some properties of Green's functions that are used to define an operator. In Sect. 3, the existence of solution for (1.1), (1.2) is established by using the fixed point theory in cones. In Sect. 4, the main results are illustrated by an example.

\section{Some preliminaries and lemmas}

Let $\mathrm{AC}_{\delta}^{n}(J, \mathbb{R})=\left\{y: J \rightarrow \mathbb{R}, \delta^{n-1} y \in \mathrm{AC}(J, \mathbb{R})\right\}$, where $\delta=t \frac{d}{d t}$ and $\mathrm{AC}(J, \mathbb{R})$ is the space of absolutely continuous functions from $J$ into $\mathbb{R}$.

Definition 2.1 ([1]) The Hadamard fractional integral of order $\alpha>0$ for a function $g \in$ $L^{1}(J, \mathbb{R})$ is defined as

$$
{ }^{H} I^{\alpha} g(t)=\frac{1}{\Gamma(\alpha)} \int_{1}^{t}\left(\log \frac{t}{s}\right)^{\alpha-1} g(s) \frac{d s}{s},
$$

where $\log (\cdot)=\log _{e}(\cdot)$.

Definition 2.2 ([1]) The Hadamard derivative of fractional order $\alpha>0$ for a function $g \in \mathrm{AC}_{\delta}^{n}(J, \mathbb{R})$ is defined as

$$
{ }^{H} D^{\alpha} g(t)=\delta^{n}\left({ }^{H} I^{n-\alpha} g\right)(t)=\frac{1}{\Gamma(n-\alpha)}\left(t \frac{d}{d t}\right)^{n} \int_{1}^{t}\left(\log \frac{t}{s}\right)^{n-\alpha-1} g(s) \frac{d s}{s},
$$

where $n-1<\alpha<n, n=[\alpha]+1,[\alpha]$ denotes the integer part of the real number $\alpha$.

Lemma 2.1 ([1]) If $\beta-1>\gamma>0$, then

(1) ${ }^{H} I^{\gamma} \log (t)^{\beta-1}=\frac{\Gamma(\beta)}{\Gamma(\beta+\gamma)}(\log t)^{\beta+\gamma-1}$,

(2) ${ }^{H} D^{\gamma} \log (t)^{\beta-1}=\frac{\Gamma(\beta)}{\Gamma(\beta-\gamma)}(\log t)^{\beta-\gamma-1}$.

Lemma 2.2 ([1]) For $\alpha>0, n=[\alpha]+1$ and $x \in C(J) \cap L^{1}(J)$, the solution of Hadamard fractional differential equation ${ }^{H} D^{\alpha} x(t)=0$ is $x(t)=\sum_{i=1}^{n} c_{i}(\log t)^{\alpha-i}$, where $c_{i} \in \mathbb{R}(i=$ $1,2, \ldots, n)$.

Lemma 2.3 ([1]) Let $\alpha>0$. If $u \in L^{1}(J)$, then the equality ${ }^{H} D^{\alpha}\left({ }^{H} I^{\alpha} u\right)(t)=u(t)$ holds a.e. on $J$.

Lemma 2.4 ([1]) If $u \in C(J)$ and ${ }^{H} D^{\alpha} u \in L^{1}(J)$, then

$$
{ }^{H} I^{\alpha}\left({ }^{H} D^{\alpha} u\right)(t)=u(t)+c_{1}(\log t)^{\alpha-1}+c_{2}(\log t)^{\alpha-2}+\cdots+c_{n}(\log t)^{\alpha-n},
$$

where $c_{i} \in \mathbb{R}(i=1,2,3 \ldots, n), n=[\alpha]+1$.

For further analysis, we introduce the following denotations:

$$
\begin{aligned}
& l_{1}=\frac{1}{\Gamma(\alpha)} \int_{1}^{+\infty} g_{1}(t)(\log t)^{\alpha-1} \frac{d t}{t}, \quad l_{2}=\frac{1}{\Gamma(\alpha-1)} \int_{1}^{+\infty} g_{2}(t)(\log t)^{\alpha-2} \frac{d t}{t}, \\
& \delta_{1}=\frac{1}{\Gamma(\alpha-1)} \int_{1}^{+\infty} g_{1}(t)(\log t)^{\alpha-2} \frac{d t}{t}, \quad \delta_{2}=\frac{1}{\Gamma(\alpha)} \int_{1}^{+\infty} g_{2}(t)(\log t)^{\alpha-1} \frac{d t}{t},
\end{aligned}
$$




$$
\begin{aligned}
& \delta=\frac{1}{\left(1-\delta_{1}\right)\left(1-\delta_{2}\right)-l_{1} l_{2}}, \quad \varphi_{\lambda}(t)=\frac{(\log t)^{\lambda-1}}{\Gamma(\lambda)}, \\
& G_{\lambda}(t, s)=\frac{1}{\Gamma(\lambda)} \begin{cases}(\log t)^{\lambda-1}-\left(\log \frac{t}{s}\right)^{\lambda-1}, & 1 \leq s \leq t<+\infty, \\
(\log t)^{\lambda-1}, & 1 \leq t \leq s<+\infty\end{cases} \\
& T_{1, \lambda}(t)=\varphi_{\lambda}(t) \delta l_{2}+\varphi_{\lambda-1}(t) \delta\left(1-\delta_{2}\right), \quad T_{2, \lambda}(t)=\varphi_{\lambda}(t) \delta\left(1-\delta_{1}\right)+\varphi_{\lambda-1}(t) \delta l_{1}, \\
& K_{\lambda}(t, s)=G_{\lambda}(t, s)+T_{1, \lambda}(t) \int_{1}^{\infty} g_{1}(t) G_{\alpha}(t, s) \frac{d t}{t}+T_{2, \lambda}(t) \int_{1}^{\infty} g_{2}(t) G_{\alpha}(t, s) \frac{d t}{t} .
\end{aligned}
$$

We will use the following conditions:

$\left(\mathbf{H}_{1}\right) q \in C\left(J, \mathbb{R}^{+}\right), f: J \times \mathbb{R}^{3} \rightarrow \mathbb{R}$ satisfies $q$-Carathéodory condition;

$\left(\mathbf{H}_{2}\right) g_{1}, g_{2} \in L^{1}\left(J, \mathbb{R}^{+}\right), l_{1}, \delta_{2}<+\infty$, and $\left(1-\delta_{1}\right)\left(1-\delta_{2}\right)-l_{1} l_{2}>0$.

Lemma 2.5 Let $h \in C(J) \cap L^{1}(J)$ with $0<\int_{1}^{\infty} h(s) \frac{d s}{s}<\infty$, then the solution of Hadamard type fractional differential equation

$$
{ }^{H} D^{\alpha} u(t)+h(t)=0,
$$

subject to the same condition (1.2) can be expressed by

$$
u(t)=\int_{1}^{+\infty} K_{\alpha}(t, s) h(s) \frac{d s}{s}
$$

where $K_{\alpha}(t, s)$ is denotation (2.3) with $\lambda=\alpha$.

Proof Due to Lemma 2.4, the solution of Hadamard fractional differential equation ${ }^{H} D^{\alpha} u(t)+h(t)=0$ can be written as

$$
u(t)=-{ }^{H} I^{\alpha} h(t)+c_{1}(\log t)^{\alpha-1}+c_{2}(\log t)^{\alpha-2}+c_{3}(\log t)^{\alpha-3},
$$

where $c_{i} \in \mathbb{R}(i=1,2,3)$ are arbitrary constants. From $u(1)=0$, we have $c_{3}=0$. By Lemma 2.1, we have

$$
{ }^{H} D^{\alpha-2} u(t)=-{ }^{H} I^{2} h(t)+c_{1} \frac{\Gamma(\alpha)}{\Gamma(2)}(\log t)+c_{2} \frac{\Gamma(\alpha-1)}{\Gamma(1)} .
$$

Using the condition ${ }^{H} D^{\alpha-2} u(1)=\int_{1}^{+\infty} g_{1}(s) u(s) \frac{d s}{s}$, we conclude that $c_{2}=\frac{1}{\Gamma(\alpha-1)} \int_{1}^{+\infty} g_{1}(t) \times$ $u(t) \frac{d t}{t}$.

Similarly, we get

$$
{ }^{H} D^{\alpha-1} u(t)=-{ }^{H} I^{1} h(t)+c_{1} \frac{\Gamma(\alpha)}{\Gamma(1)} .
$$

From the condition ${ }^{H} D^{\alpha-1} u(+\infty)=\int_{1}^{+\infty} g_{2}(s) u(s) \frac{d s}{s}$, we conclude that

$$
c_{1}=\frac{1}{\Gamma(\alpha)} \int_{1}^{+\infty} h(t) \frac{d t}{t}+\frac{1}{\Gamma(\alpha)} \int_{1}^{+\infty} g_{2}(t) u(t) \frac{d t}{t} .
$$


Consequently,

$$
\begin{aligned}
u(t)= & -{ }^{H} I^{\alpha} h(t)+c_{1}(\log t)^{\alpha-1}+c_{2}(\log t)^{\alpha-2} \\
= & -\frac{1}{\Gamma(\alpha)} \int_{1}^{t}\left(\log \frac{t}{s}\right)^{\alpha-1} h(s) \frac{d s}{s}+\frac{1}{\Gamma(\alpha)} \int_{1}^{\infty}(\log t)^{\alpha-1} h(s) \frac{d s}{s} \\
& +\frac{(\log t)^{\alpha-1}}{\Gamma(\alpha)} \int_{1}^{\infty} g_{2}(s) u(s) \frac{d s}{s}+\frac{(\log t)^{\alpha-2}}{\Gamma(\alpha-1)} \int_{1}^{\infty} g_{1}(s) u(s) \frac{d s}{s} \\
= & \int_{1}^{\infty} G_{\alpha}(t, s) h(s) \frac{d s}{s}+\frac{(\log t)^{\alpha-1}}{\Gamma(\alpha)} \int_{1}^{\infty} g_{2}(s) u(s) \frac{d s}{s} \\
& +\frac{(\log t)^{\alpha-2}}{\Gamma(\alpha-1)} \int_{1}^{\infty} g_{1}(s) u(s) \frac{d s}{s} .
\end{aligned}
$$

Multiplying both sides of (2.6) by $\frac{g_{1}(t)}{t}$ and integrating from 1 to $+\infty$, we get

$$
\begin{aligned}
\int_{1}^{+\infty} u(t) g_{1}(t) \frac{d t}{t}= & \int_{1}^{+\infty} g_{1}(t)\left(\int_{1}^{\infty} G_{\alpha}(t, s) h(s) \frac{d s}{s}\right) \frac{d t}{t} \\
& +\frac{1}{\Gamma(\alpha)} \int_{1}^{+\infty} g_{1}(t)(\log t)^{\alpha-1} \frac{d t}{t} \int_{1}^{\infty} g_{2}(s) u(s) \frac{d s}{s} \\
& +\frac{1}{\Gamma(\alpha-1)} \int_{1}^{+\infty} g_{1}(t)(\log t)^{\alpha-2} \frac{d t}{t} \int_{1}^{\infty} g_{1}(s) u(s) \frac{d s}{s}
\end{aligned}
$$

Similarly,

$$
\begin{aligned}
\int_{1}^{+\infty} u(t) g_{2}(t) \frac{d t}{t}= & \int_{1}^{+\infty} g_{2}(t)\left(\int_{1}^{\infty} G_{\alpha}(t, s) h(s) \frac{d s}{s}\right) \frac{d t}{t} \\
& +\frac{1}{\Gamma(\alpha)} \int_{1}^{+\infty} g_{2}(t)(\log t)^{\alpha-1} \frac{d t}{t} \int_{1}^{\infty} g_{2}(s) u(s) \frac{d s}{s} \\
& +\frac{1}{\Gamma(\alpha-1)} \int_{1}^{+\infty} g_{2}(t)(\log t)^{\alpha-2} \frac{d t}{t} \int_{1}^{\infty} g_{1}(s) u(s) \frac{d s}{s}
\end{aligned}
$$

For convenience, we denote

$$
\begin{aligned}
& X_{1}=\int_{1}^{+\infty} g_{1}(t) u(t) \frac{d t}{t}, \quad X_{2}=\int_{1}^{+\infty} g_{2}(t) u(t) \frac{d t}{t}, \\
& A_{1}=\int_{1}^{+\infty}\left(\int_{1}^{\infty} g_{1}(t) G_{\alpha}(t, s) \frac{d t}{t}\right) h(s) \frac{d s}{s}, \quad A_{2}=\int_{1}^{+\infty}\left(\int_{1}^{\infty} g_{2}(t) G_{\alpha}(t, s) \frac{d t}{t}\right) h(s) \frac{d s}{s} .
\end{aligned}
$$

From (2.7), (2.8) we can deduce

$$
\left(\begin{array}{cc}
1-\delta_{1} & -l_{1} \\
-l_{2} & 1-\delta_{2}
\end{array}\right)\left(\begin{array}{l}
X_{1} \\
X_{2}
\end{array}\right)=\left(\begin{array}{l}
A_{1} \\
A_{2}
\end{array}\right)
$$

Thus,

$$
\begin{aligned}
& X_{1}=\frac{\left(1-\delta_{2}\right) A_{1}+l_{1} A_{2}}{\left(1-\delta_{1}\right)\left(1-\delta_{2}\right)-l_{1} l_{2}}=\delta\left(\left(1-\delta_{2}\right) A_{1}+l_{1} A_{2}\right), \\
& X_{2}=\frac{l_{2} A_{1}+\left(1-\delta_{1}\right) A_{2}}{\left(1-\delta_{1}\right)\left(1-\delta_{2}\right)-l_{1} l_{2}}=\delta\left(l_{2} A_{1}+\left(1-\delta_{1}\right) A_{2}\right) .
\end{aligned}
$$


Substituting $X_{1}, X_{2}$ into (2.6), we can conclude

$$
\begin{aligned}
u(t)= & \int_{1}^{\infty} G_{\alpha}(t, s) h(s) \frac{d s}{s}+\frac{(\log t)^{\alpha-1}}{\Gamma(\alpha)} \delta\left(l_{2} A_{1}+\left(1-\delta_{1}\right) A_{2}\right) \\
& +\frac{(\log t)^{\alpha-2}}{\Gamma(\alpha-1)} \delta\left(\left(1-\delta_{2}\right) A_{1}+l_{1} A_{2}\right) \\
= & \int_{1}^{\infty} G_{\alpha}(t, s) h(s) \frac{d s}{s}+\left[\frac{(\log t)^{\alpha-1}}{\Gamma(\alpha)} \delta l_{2}+\frac{(\log t)^{\alpha-2}}{\Gamma(\alpha-1)} \delta\left(1-\delta_{2}\right)\right] A_{1} \\
& +\left[\frac{(\log t)^{\alpha-1}}{\Gamma(\alpha)} \delta\left(1-\delta_{1}\right)+\frac{(\log t)^{\alpha-2}}{\Gamma(\alpha-1)} \delta l_{1}\right] A_{2} \\
= & \int_{1}^{\infty} G_{\alpha}(t, s) h(s) \frac{d s}{s}+T_{1, \alpha}(t) A_{1}+T_{2, \alpha}(t) A_{2} \\
= & \int_{1}^{\infty}\left[G_{\alpha}(t, s)+T_{1, \alpha}(t) \int_{1}^{\infty} g_{1}(t) G_{\alpha}(t, s) \frac{d t}{t}+T_{2, \alpha}(t) \int_{1}^{\infty} g_{2}(t) G_{\alpha}(t, s) \frac{d t}{t}\right] h(s) \frac{d s}{s} \\
= & \int_{1}^{\infty} K_{\alpha}(t, s) h(s) \frac{d s}{s} .
\end{aligned}
$$

Lemma 2.6 The functions $G_{\lambda}, \varphi_{\lambda}, T_{1, \lambda}, T_{2, \lambda}$ defined in (2.1), (2.2) satisfy

(1) $G_{\lambda}$ is continuous in $J \times J$ and $0 \leq G_{\lambda}(t, s) \leq \frac{1}{\Gamma(\lambda)}(\log t)^{\lambda-1}, \forall t, s \in J \times J, \lambda \geq 1$;

(2) $\frac{G_{\lambda}(t, s)}{1+(\log t)^{\lambda-1}} \leq \frac{1}{\Gamma(\lambda)}, \forall t, s \in J \times J, \lambda \geq 1$;

(3) If $\lambda \geq 2$, for any $t \in J, \frac{\varphi_{\lambda}(t)}{1+(\log t)^{\lambda-1}} \leq \frac{1}{\Gamma(\lambda)}, \frac{\varphi_{\lambda-1}(t)}{1+(\log t)^{\lambda-1}} \leq \frac{1}{\Gamma(\lambda-1)}$;

(4) If $1<\lambda<2$, for any $t, s \in J$,

$$
\begin{aligned}
\frac{(\log t)^{2-\lambda}}{1+(\log t)^{\lambda}} G_{\lambda}(t, s) & \leq \frac{1}{\Gamma(\lambda)}, \quad \frac{(\log t)^{2-\lambda}}{1+(\log t)^{\lambda}} \varphi_{\lambda}(t) \leq \frac{1}{\Gamma(\lambda)}, \\
\frac{(\log t)^{2-\lambda}}{1+(\log t)^{\lambda}} \varphi_{\lambda-1}(t) & \leq \frac{1}{\Gamma(\lambda-1)} ;
\end{aligned}
$$

(5) For any $t \in J, \lambda \geq 2$,

$$
\frac{T_{1, \lambda}(t)}{1+(\log t)^{\lambda-1}} \leq \frac{\delta l_{2}}{\Gamma(\lambda)}+\frac{\delta\left(1-\delta_{2}\right)}{\Gamma(\lambda-1)}, \quad \frac{T_{2, \lambda}(t)}{1+(\log t)^{\lambda-1}} \leq \frac{\delta\left(1-\delta_{1}\right)}{\Gamma(\lambda)}+\frac{\delta l_{1}}{\Gamma(\lambda-1)} .
$$

Proof By the definition, conclusions (1) and (2) can be easily obtained.

(3) If $\lambda \geq 2$, for $\forall t \in J, \frac{\varphi_{\lambda}(t)}{1+(\log t)^{\lambda-1}}=\frac{(\log t)^{\lambda-1}}{1+(\log t)^{\lambda-1}} \frac{1}{\Gamma(\lambda)} \leq \frac{1}{\Gamma(\lambda)}$,

$$
\begin{aligned}
\frac{\varphi_{\lambda-1}(t)}{1+(\log t)^{\lambda-1}} & =\frac{(\log t)^{\lambda-2}}{1+(\log t)^{\lambda-1}} \frac{1}{\Gamma(\lambda-1)} \\
& \leq \begin{cases}\frac{(\log t)^{\lambda-1}}{1+(\log t)^{\lambda-1}} \frac{1}{\Gamma(\lambda-1)}, \quad t \geq e, \\
\frac{1}{1+(\log t)^{\lambda-1}} \frac{1}{\Gamma(\lambda-1)}, \quad 1 \leq t \leq e\end{cases} \\
& \leq \frac{1}{\Gamma(\lambda-1)} .
\end{aligned}
$$

(4) If $1<\lambda<2$, for any $t, s \in J, \frac{(\log t)^{2-\lambda}}{1+(\log t)^{\lambda}} G_{\lambda}(t, s) \leq \frac{\log t}{1+(\log t)^{\lambda}} \frac{1}{\Gamma(\lambda)} \leq \frac{1}{\Gamma(\lambda)}$,

$$
\frac{(\log t)^{2-\lambda}}{1+(\log t)^{\lambda}} \varphi_{\lambda}(t)=\frac{\log t}{1+(\log t)^{\lambda}} \frac{1}{\Gamma(\lambda)} \leq \frac{1}{\Gamma(\lambda)}
$$




$$
\frac{(\log t)^{2-\lambda}}{1+(\log t)^{\lambda}} \varphi_{\lambda-1}(t)=\frac{1}{1+(\log t)^{\lambda}} \frac{1}{\Gamma(\lambda-1)} \leq \frac{1}{\Gamma(\lambda-1)}
$$

On the basis of (3), conclusion (5) can be easily deduced.

Remark 1 From Lemma 2.1 and $0<\beta_{1} \leq \alpha-2<\beta_{2} \leq \alpha-1$, we can calculate

$$
\begin{aligned}
& { }^{H} D^{\beta_{1}} T_{1, \alpha}(t)=T_{1, \alpha-\beta_{1}}(t), \quad{ }^{H} D^{\beta_{1}} T_{2, \alpha}(t)=T_{2, \alpha-\beta_{1}}(t), \\
& { }^{H} D^{\beta_{2}} T_{1, \alpha}(t)= \begin{cases}T_{1, \alpha-\beta_{2}}(t), & \beta_{2}<\alpha-1, \\
\delta l_{2}, & \beta_{2}=\alpha-1,\end{cases} \\
& { }^{H} D^{\beta_{2}} T_{2, \alpha}(t)= \begin{cases}T_{2, \alpha-\beta_{2}}(t), & \beta_{2}<\alpha-1, \\
\delta\left(1-\delta_{1}\right), & \beta_{2}=\alpha-1 .\end{cases}
\end{aligned}
$$

Define the function spaces

$$
X=\left\{u \in C(J): \sup _{t \in J} \frac{|u(t)|}{1+(\log t)^{\alpha-1}}<+\infty\right\}
$$

with the form $\|u\|_{X}=\sup _{t \in J} \frac{|u(t)|}{1+(\log t)^{\alpha-1}}$ and

$$
\begin{aligned}
Y= & \left\{u \in X:{ }^{H} D^{\beta_{1}} u \in C(J),{ }^{H} D^{\beta_{2}} u \in C(1,+\infty), \sup _{t \in J} \frac{\left|{ }^{H} D^{\beta_{1}} u(t)\right|}{1+(\log t)^{\alpha-\beta_{1}-1}}<+\infty,\right. \\
& \left.\sup _{t \in J} \frac{(\log t)^{2+\beta_{2}-\alpha}}{1+(\log t)^{\alpha-\beta_{2}}}\left|{ }^{H} D^{\beta_{2}} u(t)\right|<+\infty\right\}
\end{aligned}
$$

with the norm

$$
\|u\|_{Y}=\max \left\{\sup _{t \in J} \frac{|u(t)|}{1+(\log t)^{\alpha-1}}, \sup _{t \in J} \frac{\left|{ }^{H} D^{\beta_{1}} u(t)\right|}{1+(\log t)^{\alpha-\beta_{1}-1}}, \sup _{t \in J} \frac{(\log t)^{2+\beta_{2}-\alpha}}{1+(\log t)^{\alpha-\beta_{2}}}\left|{ }^{H} D^{\beta_{2}} u(t)\right|\right\} .
$$

By a standard method, we can show that $\left(Y,\|\cdot\|_{Y}\right)$ is a Banach space.

According to the same method in $[46,49]$, we can get the following lemma.

Lemma 2.7 Let $U \subset Y$ be a bounded set, then $U$ is relatively compact in $Y$ if the following conditions hold:

(1) For any $u \in U, \frac{u(t)}{1+(\log t)^{\alpha-1}}, \frac{H_{D} D_{1} u(t)}{1+(\log t)^{\alpha-\beta} 1^{-1}}$, and $\frac{(\log t)^{2+\beta_{2}-\alpha}}{1+(\log t)^{\alpha-\beta_{2}}}\left({ }^{H} D^{\beta_{2}} u\right)(t)$ are equicontinuous on any compact interval of $J$;

(2) For any $\varepsilon>0$, there exists a constant $L=L(\varepsilon)>0$ such that

$$
\left|\frac{u\left(t_{1}\right)}{1+\left(\log t_{1}\right)^{\alpha-1}}-\frac{u\left(t_{2}\right)}{1+\left(\log t_{2}\right)^{\alpha-1}}\right|<\varepsilon, \quad\left|\frac{{ }^{H} D^{\beta_{1}} u\left(t_{1}\right)}{1+\left(\log t_{1}\right)^{\alpha-\beta_{1}-1}}-\frac{{ }^{H} D^{\beta_{1}} u\left(t_{2}\right)}{1+\left(\log t_{2}\right)^{\alpha-\beta_{1}-1}}\right|<\varepsilon,
$$

and

$$
\left|\frac{\left(\log t_{1}\right)^{2+\beta_{2}-\alpha}}{1+\left(\log t_{1}\right)^{\alpha-\beta_{2}}}\left({ }^{H} D^{\beta_{2}} u\right)\left(t_{1}\right)-\frac{\left(\log t_{2}\right)^{2+\beta_{2}-\alpha}}{1+\left(\log t_{2}\right)^{\alpha-\beta_{2}}}\left({ }^{H} D^{\beta_{2}} u\right)\left(t_{2}\right)\right|<\varepsilon,
$$

for any $t_{1}, t_{2} \geq L$ and $u \in U$. 
Lemma 2.8 (Schauder's fixed point theorem) Let C be a compact, closed, bounded, and convex subset of a Banach space X. Suppose that $T: C \rightarrow C$ is a continuous and compact mapping. Then $T$ has at least one fixed point in $C$.

\section{Main results}

In this section, we shall establish the existence result of at least one solution of (1.1), (1.2). For convenience, we denote $F_{u}(s)=f\left(s, u(s),{ }^{H} D^{\beta_{1}} u(s),{ }^{H} D^{\beta_{2}} u(s)\right)$.

We define an operator $A: Y \rightarrow C(J, \mathbb{R})$ as follows:

$$
A u(t)=\int_{1}^{+\infty} K_{\alpha}(t, s) q(s) F_{u}(s) \frac{d s}{s}, \quad t \in J
$$

By Lemma 2.5, we can show that $u(t)$ is a solution of boundary value problem (1.1), (1.2) if and only if it is the fixed point of $A$.

From Lemma 2.1 and Remark 1, we have

$$
\begin{aligned}
{ }^{H} D^{\beta_{1}} A u(t)= & { }^{H} D^{\beta_{1}}\left(\int_{1}^{+\infty} K_{\alpha}(t, s) q(s) F_{u}(s) \frac{d s}{s}\right) \\
= & D^{\beta_{1}}\left(-\frac{1}{\Gamma(\alpha)} \int_{1}^{t}\left(\log \frac{t}{s}\right)^{\alpha-1} q(s) F_{u}(s) \frac{d s}{s}+\frac{(\log t)^{\alpha-1}}{\Gamma(\alpha)} \int_{1}^{\infty} q(s) F_{u}(s) \frac{d s}{s}\right. \\
& +T_{1, \alpha}(t) \int_{1}^{\infty}\left(\int_{1}^{\infty} g_{1}(t) G_{\alpha}(t, s) \frac{d t}{t}\right) q(s) F_{u}(s) \frac{d s}{s} \\
& \left.+T_{2, \alpha}(t) \int_{1}^{\infty}\left(\int_{1}^{\infty} g_{2}(t) G_{\alpha}(t, s) \frac{d t}{t}\right) q(s) F_{u}(s) \frac{d s}{s}\right) \\
= & -{ }^{H} I^{\alpha-\beta_{1}} q(t) F_{u}(t)+\frac{(\log t)^{\alpha-\beta_{1}-1}}{\Gamma\left(\alpha-\beta_{1}\right)} \int_{1}^{\infty} q(s) F_{u}(s) \frac{d s}{s} \\
& +T_{1, \alpha-\beta_{1}}(t) \int_{1}^{\infty}\left(\int_{1}^{\infty} g_{1}(t) G_{\alpha}(t, s) \frac{d t}{t}\right) q(s) F_{u}(s) \frac{d s}{s} \\
& +T_{2, \alpha-\beta_{1}}(t) \int_{1}^{\infty}\left(\int_{1}^{\infty} g_{2}(t) G_{\alpha}(t, s) \frac{d t}{t}\right) q(s) F_{u}(s) \frac{d s}{s} \\
= & \int_{1}^{+\infty} K_{\alpha-\beta_{1}}(t, s) q(s) F_{u}(s) \frac{d s}{s} .
\end{aligned}
$$

Similarly, if $\beta_{2}<\alpha-1$,

$$
{ }^{H} D^{\beta_{2}} A u(t)=\int_{1}^{+\infty} K_{\alpha-\beta_{2}}(t, s) q(s) F_{u}(s) \frac{d s}{s}
$$

if $\beta_{2}=\alpha-1$,

$$
\begin{aligned}
{ }^{H} D^{\beta_{2}} A u(t)= & { }^{H} D^{\beta_{2}}\left(\int_{1}^{+\infty} K_{\alpha}(t, s) q(s) F_{u}(s) \frac{d s}{s}\right) \\
= & { }^{H} D^{\beta_{2}}\left(-\frac{1}{\Gamma(\alpha)} \int_{1}^{t}\left(\log \frac{t}{s}\right)^{\alpha-1} q(s) F_{u}(s) \frac{d s}{s}+\frac{(\log t)^{\alpha-1}}{\Gamma(\alpha)} \int_{1}^{\infty} q(s) F_{u}(s) \frac{d s}{s}\right. \\
& +T_{1, \alpha}(t) \int_{1}^{\infty}\left(\int_{1}^{\infty} g_{1}(t) G_{\alpha}(t, s) \frac{d t}{t}\right) q(s) F_{u}(s) \frac{d s}{s}
\end{aligned}
$$




$$
\begin{aligned}
& \left.+T_{2, \alpha}(t) \int_{1}^{\infty}\left(\int_{1}^{\infty} g_{2}(t) G_{\alpha}(t, s) \frac{d t}{t}\right) q(s) F_{u}(s) \frac{d s}{s}\right) \\
= & -{ }^{H} I^{1} q(t) F_{u}(t)+\int_{1}^{\infty} q(s) F_{u}(s) \frac{d s}{s} \\
& +\delta l_{2} \int_{1}^{\infty}\left(\int_{1}^{\infty} g_{1}(t) G_{\alpha}(t, s) \frac{d t}{t}\right) q(s) F_{u}(s) \frac{d s}{s} \\
& +\delta\left(1-\delta_{1}\right) \int_{1}^{\infty}\left(\int_{1}^{\infty} g_{2}(t) G_{\alpha}(t, s) \frac{d t}{t}\right) q(s) F_{u}(s) \frac{d s}{s} \\
= & \int_{1}^{+\infty} K^{\beta_{2}}(t, s) q(s) F_{u}(s) \frac{d s}{s},
\end{aligned}
$$

where $K^{\beta_{2}}(t, s)=G(t, s)+\delta l_{2} \int_{1}^{\infty} g_{1}(t) G_{\alpha}(t, s) \frac{d t}{t}+\delta\left(1-\delta_{1}\right) \int_{1}^{\infty} g_{2}(t) G_{\alpha}(t, s) \frac{d t}{t}$,

$$
G(t, s)= \begin{cases}0, & 1 \leq s \leq t<+\infty \\ 1, & 1 \leq t \leq s<+\infty\end{cases}
$$

Remark 2 In (2.3) we choose $\lambda=\alpha, \lambda=\alpha-\beta_{1}$ respectively, from Lemma 2.6, we can easily get the following inequalities:

$$
\begin{aligned}
\frac{K_{\alpha}(t, s)}{1+(\log t)^{\alpha-1}} \leq & \frac{1}{\Gamma(\alpha)}+l_{1}\left(\frac{\delta l_{2}}{\Gamma(\alpha)}+\frac{\delta\left(1-\delta_{2}\right)}{\Gamma(\alpha-1)}\right) \\
& +\delta_{2}\left(\frac{\delta\left(1-\delta_{1}\right)}{\Gamma(\alpha)}+\frac{\delta l_{1}}{\Gamma(\alpha-1)}\right) \\
\triangleq & \Delta_{1}, \quad \forall t, s \in J, \\
\frac{K_{\alpha-\beta_{1}}(t, s)}{1+(\log t)^{\alpha-\beta_{1}-1}} \leq & \frac{1}{\Gamma\left(\alpha-\beta_{1}\right)}+l_{1}\left(\frac{\delta l_{2}}{\Gamma\left(\alpha-\beta_{1}\right)}+\frac{\delta\left(1-\delta_{2}\right)}{\Gamma\left(\alpha-\beta_{1}-1\right)}\right) \\
& +\delta_{2}\left(\frac{\delta\left(1-\delta_{1}\right)}{\Gamma\left(\alpha-\beta_{1}\right)}+\frac{\delta l_{1}}{\Gamma\left(\alpha-\beta_{1}-1\right)}\right) \\
\triangleq & \Delta_{2}, \quad \forall t, s \in J
\end{aligned}
$$

when $1<\alpha-\beta_{2}<2$, for all $t, s \in J$, according to Lemma 2.6(2), (4), let $\lambda=\alpha-\beta_{2}$,

$$
\begin{aligned}
\frac{(\log t)^{2+\beta_{2}-\alpha}}{1+(\log t)^{\alpha-\beta_{2}}} K_{\alpha-\beta_{2}}(t, s)= & \frac{(\log t)^{2+\beta_{2}-\alpha}}{1+(\log t)^{\alpha-\beta_{2}}} G_{\alpha-\beta_{2}}(t, s) \\
& +\frac{(\log t)^{2+\beta_{2}-\alpha}}{1+(\log t)^{\alpha-\beta_{2}}} T_{1, \alpha-\beta_{2}}(t) \int_{1}^{+\infty} g_{1}(s) G_{\alpha}(t, s) \frac{d t}{t} \\
& +\frac{(\log t)^{2+\beta_{2}-\alpha}}{1+(\log t)^{\alpha-\beta_{2}}} T_{2, \alpha-\beta_{2}}(t) \int_{1}^{+\infty} g_{2}(s) G_{\alpha}(t, s) \frac{d t}{t} \\
\leq & \frac{1}{\Gamma\left(\alpha-\beta_{2}\right)}+\left[\frac{\delta l_{2}}{\Gamma\left(\alpha-\beta_{2}\right)}+\frac{\delta\left(1-\delta_{2}\right)}{\Gamma\left(\alpha-\beta_{2}-1\right)}\right] l_{1} \\
& +\left[\frac{\delta\left(1-\delta_{1}\right)}{\Gamma\left(\alpha-\beta_{2}\right)}+\frac{\delta l_{1}}{\Gamma\left(\alpha-\beta_{2}-1\right)}\right] \delta_{2} \\
\triangleq & \Delta_{3}
\end{aligned}
$$


when $\alpha-\beta_{2}=1$, we have the following inequality:

$$
\frac{(\log t)^{2+\beta_{2}-\alpha}}{1+(\log t)^{\alpha-\beta_{2}}} K^{\beta_{2}}(t, s)=\frac{\log t}{1+\log t} K^{\beta_{2}}(t, s) \leq 1+\delta l_{1} l_{2}+\delta\left(1-\delta_{1}\right) \delta_{2} \triangleq \Delta_{4} .
$$

Lemma 3.1 The operator $A: Y \rightarrow Y$ is well defined.

Proof For any $u \in Y, u \neq \theta$, let $\|u\|_{Y}=r>0$. In view of the definition of $Y$,

$$
\begin{aligned}
& \sup _{t \in J} \frac{|u(t)|}{1+(\log t)^{\alpha-1}} \leq r, \quad \sup _{t \in J} \frac{\left|{ }^{H} D^{\beta_{1}} u(t)\right|}{1+(\log t)^{\alpha-\beta_{1}-1}} \leq r, \\
& \left.\sup _{t \in J} \frac{(\log t)^{2+\beta_{2}-\alpha}}{1+(\log t)^{\alpha-\beta_{2}}}\right|^{H} D^{\beta_{2}} u(t) \mid \leq r .
\end{aligned}
$$

From the continuity of $K_{\alpha}, q, F_{u}$, we know $A u(t),{ }^{H} D^{\beta_{1}} A u(t)$, and ${ }^{H} D^{\beta_{2}} A u(t)$ are continuous.

$$
\begin{aligned}
\left|F_{u}(s)\right|= & \left|f\left(s, u(s),{ }^{H} D^{\beta_{1}} u(s),{ }^{H} D^{\beta_{2}} u(s)\right)\right| \\
= & \mid f\left(s,\left(1+(\log s)^{\alpha-1}\right) \frac{u(s)}{1+(\log s)^{\alpha-1}},\left(1+(\log s)^{\alpha-\beta_{1}-1}\right) \frac{{ }^{H} D^{\beta_{1}} u(s)}{1+(\log s)^{\alpha-\beta_{1}-1}},\right. \\
& \left.\frac{1+(\log s)^{\alpha-\beta_{2}}}{(\log s)^{2+\beta_{2}-\alpha}} \frac{(\log s)^{2+\beta_{2}-\alpha}}{1+(\log s)^{\alpha-\beta_{2}}}{ }^{H} D^{\beta_{2}} u(s)\right) \mid \\
\leq & f_{r}(s) .
\end{aligned}
$$

Using (3.5)-(3.8) and the Caratheodory condition, we can deduce

$$
\begin{aligned}
& \sup _{t \in J}\left|\frac{A u(t)}{1+(\log t)^{\alpha-1}}\right|=\sup _{t \in J}\left|\int_{1}^{+\infty} \frac{K_{\alpha}(t, s)}{1+(\log t)^{\alpha-1}} q(s) F_{u}(s) \frac{d s}{s}\right| \leq \Delta_{1} \int_{1}^{+\infty} q(s) f_{r}(s) \frac{d s}{s}<+\infty \text {, } \\
& \sup _{t \in J}\left|\frac{{ }^{H} D^{\beta_{1}} A u(t)}{1+(\log t)^{\alpha-\beta_{1}-1}}\right|=\sup _{t \in J}\left|\int_{1}^{+\infty} \frac{K_{\alpha-\beta_{1}}(t, s)}{1+(\log t)^{\alpha-\beta_{1}-1}} q(s) F_{u}(s) \frac{d s}{s}\right| \\
& \leq \Delta_{2} \int_{1}^{+\infty} q(s) f_{r}(s) \frac{d s}{s}<+\infty, \\
& \sup _{t \in J}\left|\frac{(\log t)^{2+\beta_{2}-\alpha}}{1+(\log t)^{\alpha-\beta_{2}}}\left({ }^{H} D^{\beta_{2}} A u_{n}\right)(t)\right|=\sup _{t \in J}\left|\int_{1}^{+\infty} \frac{(\log t)^{2+\beta_{2}-\alpha}}{1+(\log t)^{\alpha-\beta_{2}}} K_{\alpha-\beta_{2}}(t, s) q(s) F_{u}(s) \frac{d s}{s}\right| \\
& \leq \Delta_{3} \int_{1}^{+\infty} q(s) f_{r}(s) \frac{d s}{s}<+\infty, \quad\left(\beta_{2}<\alpha-1\right),
\end{aligned}
$$

and

$$
\begin{aligned}
\sup _{t \in J}\left|\frac{(\log t)^{2+\beta_{2}-\alpha}}{1+(\log t)^{\alpha-\beta_{2}}}\left({ }^{H} D^{\beta_{2}} A u_{n}\right)(t)\right| & =\sup _{t \in J}\left|\int_{1}^{+\infty} \frac{(\log t)^{2+\beta_{2}-\alpha}}{1+(\log t)^{\alpha-\beta_{2}}} K^{\beta_{2}}(t, s) q(s) F_{u}(s) \frac{d s}{s}\right| \\
& \leq \Delta_{4} \int_{1}^{+\infty} q(s) f_{r}(s) \frac{d s}{s}<+\infty, \quad\left(\beta_{2}=\alpha-1\right) .
\end{aligned}
$$

From the definition of space $Y$, we know $A u \in Y$, which means operator $A$ is well defined. 
Theorem 3.1 Assume that conditions $\left(\mathbf{H}_{1}\right)-\left(\mathbf{H}_{2}\right)$ are satisfied. Further assume that the following condition $\left(\mathbf{H}_{3}\right)$ holds:

$\left(\mathbf{H}_{3}\right)$ There exists a constant $R>0$ such that $f_{R}(t)$ satisfies

$$
\Delta \int_{1}^{+\infty} q(s) f_{R}(s) \frac{d s}{s} \leq R
$$

where $\Delta=\max \left\{\Delta_{1}, \Delta_{2}, \Delta_{3}, \Delta_{4}\right\}$. Then boundary value problem (1.1), (1.2) has at least one solution.

Proof The proof is divided into the following steps:

Step 1. We will prove that $A: Y \rightarrow Y$ is completely continuous.

Firstly we will show that $A$ is a continuous operator. Let $u_{n}, u \in Y(n=1,2, \ldots)$ with $\left\|u_{n}-u\right\|_{Y} \rightarrow 0, n \rightarrow \infty$. For any $t \in J$, we have

$$
\begin{aligned}
& \frac{u_{n}(t)}{1+(\log t)^{\alpha-1}} \rightarrow \frac{u(t)}{1+(\log t)^{\alpha-1}}, \quad \frac{{ }^{H} D^{\beta_{1}} u_{n}(t)}{1+(\log t)^{\alpha-\beta_{1}-1}} \rightarrow \frac{{ }^{H} D^{\beta_{1}} u(t)}{1+(\log t)^{\alpha-\beta_{1}-1}}, \\
& \frac{(\log t)^{2+\beta_{2}-\alpha}}{1+(\log t)^{\alpha-\beta_{2}}}\left({ }^{H} D^{\beta_{2}} u_{n}(t)\right) \rightarrow \frac{(\log t)^{2+\beta_{2}-\alpha}}{1+(\log t)^{\alpha-\beta_{2}}}\left({ }^{H} D^{\beta_{2}} u(t)\right), \quad n \rightarrow \infty .
\end{aligned}
$$

Meanwhile, there exists a constant $r>0$ such that $\left\|u_{n}\right\|_{Y} \leq r,\|u\|_{Y} \leq r$, which means

$$
\begin{aligned}
& \sup _{t \in J}\left|\frac{u_{n}(t)}{1+(\log t)^{\alpha-1}}\right| \leq r, \quad \sup _{t \in J}\left|\frac{u(t)}{1+(\log t)^{\alpha-1}}\right| \leq r, \\
& \sup _{t \in J}\left|\frac{{ }^{H} D^{\beta_{1}} u_{n}(t)}{1+(\log t)^{\alpha-\beta_{1}-1}}\right| \leq r, \quad \sup _{t \in J}\left|\frac{{ }^{H} D^{\beta_{1}} u(t)}{1+(\log t)^{\alpha-\beta_{1}-1}}\right| \leq r, \\
& \sup _{t \in J}\left|\frac{(\log t)^{2+\beta_{2}-\alpha}}{1+(\log t)^{\alpha-\beta_{2}}}\left({ }^{H} D^{\beta_{2}} u_{n}\right)(t)\right| \leq r, \quad \sup _{t \in J}\left|\frac{(\log t)^{2+\beta_{2}-\alpha}}{1+(\log t)^{\alpha-\beta_{2}}}\left({ }^{H} D^{\beta_{2}} u\right)(t)\right| \leq r .
\end{aligned}
$$

Due to the Caratheodory condition, for a.e. $s \in J$, we have

$$
\left|F_{u_{n}}(s)-F_{u}(s)\right| \rightarrow 0, \quad(n \rightarrow \infty) \quad\left|F_{u_{n}}(s)-F_{u}(s)\right| \leq 2 f_{r}(s) .
$$

By the Lebesgue dominated convergence theorem, we obtain

$$
\int_{1}^{+\infty}\left|F_{u_{n}}(s)-F_{u}(s)\right| \frac{d s}{s} \rightarrow 0, \quad n \rightarrow \infty
$$

Following the above method, we can also deduce

$$
\begin{aligned}
\left|\frac{A u_{n}(t)-A u(t)}{1+(\log t)^{\alpha-1}}\right| & =\left|\int_{1}^{+\infty} \frac{K_{\alpha}(t, s)}{1+(\log t)^{\alpha-1}} q(s)\left(F_{u_{n}}(s)-F_{u}(s)\right) \frac{d s}{s}\right| \\
& \leq \Delta_{1} \int_{1}^{+\infty} q(s)\left|F_{u_{n}}(s)-F_{u}(s)\right| \frac{d s}{s} \rightarrow 0, \quad n \rightarrow+\infty, \\
\left|\frac{{ }^{H} D^{\beta_{1}} A u_{n}(t)-{ }^{H} D^{\beta_{1}} A u(t)}{1+(\log t)^{\alpha-\beta_{1}-1}}\right| & =\left|\int_{1}^{+\infty} \frac{K_{\alpha-\beta}(t, s)}{1+(\log t)^{\alpha-\beta-1}} q(s)\left(F_{u_{n}}(s)-F_{u}(s)\right) \frac{d s}{s}\right| \\
& \leq \Delta_{2} \int_{1}^{+\infty} q(s)\left|F_{u_{n}}(s)-F_{u}(s)\right| \frac{d s}{s} \rightarrow 0, \quad n \rightarrow+\infty,
\end{aligned}
$$




$$
\begin{aligned}
& \left|\frac{(\log t)^{2+\beta_{2}-\alpha}}{1+(\log t)^{\alpha-\beta_{2}}}\left({ }^{H} D^{\beta_{2}} A u_{n}\right)(t)-\frac{(\log t)^{2+\beta_{2}-\alpha}}{1+(\log t)^{\alpha-\beta_{2}}}\left({ }^{H} D^{\beta_{2}} A u\right)(t)\right| \\
& \quad \leq \Delta_{3} \int_{1}^{+\infty} q(s)\left|F_{u_{n}}(s)-F_{u}(s)\right| \frac{d s}{s} \rightarrow 0, \quad n \rightarrow+\infty(\beta<\alpha-1),
\end{aligned}
$$

and

$$
\begin{aligned}
& \left|\frac{(\log t)^{2+\beta_{2}-\alpha}}{1+(\log t)^{\alpha-\beta_{2}}}\left({ }^{H} D^{\beta_{2}} A u_{n}\right)(t)-\frac{(\log t)^{2+\beta_{2}-\alpha}}{1+(\log t)^{\alpha-\beta_{2}}}\left({ }^{H} D^{\beta_{2}} A u\right)(t)\right| \\
& \quad \leq \Delta_{4} \int_{1}^{+\infty} q(s)\left|F_{u_{n}}(s)-F_{u}(s)\right| \frac{d s}{s} \rightarrow 0, \quad n \rightarrow+\infty(\beta=\alpha-1) .
\end{aligned}
$$

Thus, we conclude $\left\|A u_{n}-A u\right\|_{Y} \rightarrow 0,(n \rightarrow+\infty)$, which means that $A: Y \rightarrow Y$ is a continuous operator.

Next, we will show that $A$ is a compact operator. Let $B$ be a nonempty bounded subset of $Y$. There exists a positive number $r_{1}$ such that $\|u\|_{Y} \leq r_{1}, \forall u \in B$, and there exists $f_{r_{1}} \in$ $L^{1}(J)$ such that $\left|F_{u}(s)\right| \leq f_{r_{1}}(s)$.

(1) For any $u \in B$, we can get

$$
\begin{aligned}
& \sup _{t \in J}\left|\frac{A u(t)}{1+(\log t)^{\alpha-1}}\right| \leq \Delta_{1} \int_{1}^{+\infty} q(s) f_{r_{1}}(s) \frac{d s}{s}<+\infty, \\
& \sup _{t \in J}\left|\frac{{ }^{H} D^{\beta_{1}} A u(t)}{1+(\log t)^{\alpha-\beta_{1}-1}}\right| \leq \Delta_{2} \int_{1}^{+\infty} q(s) f_{r_{1}}(s) \frac{d s}{s}<+\infty, \\
& \sup _{t \in J}\left|\frac{(\log t)^{2+\beta_{2}-\alpha}}{1+(\log t)^{\alpha-\beta_{2}}}\left({ }^{H} D^{\beta_{2}} A u_{n}\right)(t)\right| \leq \Delta_{3} \int_{1}^{+\infty} q(s) f_{r_{1}}(s) \frac{d s}{s}<+\infty, \quad\left(\beta_{2}<\alpha-1\right),
\end{aligned}
$$

and

$$
\sup _{t \in J}\left|\frac{(\log t)^{2+\beta_{2}-\alpha}}{1+(\log t)^{\alpha-\beta_{2}}}\left({ }^{H} D^{\beta_{2}} A u\right)(t)\right| \leq \Delta_{4} \int_{1}^{+\infty} q(s) f_{r_{1}}(s) \frac{d s}{s}<+\infty, \quad\left(\beta_{2}=\alpha-1\right)
$$

Therefore $\|A u\|_{Y} \leq \Delta \int_{1}^{+\infty} q(s) f_{r_{1}}(s) \frac{d s}{s}<+\infty$ and $A(B)$ is bounded in $Y$.

(2) For any $b>1$, let $J_{1} \triangleq[1, b]$. Because $\frac{G_{\alpha}(t, s)}{1+(\log t)^{\alpha-1}}$ is continuous on $J_{1} \times J_{1}$ and $\frac{(\log t)^{\alpha-1}}{1+(\log t)^{\alpha-1}}$, $\frac{T_{1, \alpha}(t)}{1+(\log t)^{\alpha-1}}, \frac{T_{2, \alpha}(t)}{1+(\log t)^{\alpha-1}}$ are continuous on $J_{1}$, then they are uniformly continuous. So, for any $\varepsilon>0$, there is a constant $\delta_{1}>0$ such that, for all $t_{1}, t_{2}, s_{1}, s_{2} \in J_{1}$, with $\left|t_{1}-t_{2}\right|<\delta_{1},\left|s_{1}-s_{2}\right|<$ $\delta_{1}$

$$
\begin{aligned}
& \left|\frac{G_{\alpha}\left(t_{1}, s\right)}{1+\left(\log t_{1}\right)^{\alpha-1}}-\frac{G_{\alpha}\left(t_{2}, s\right)}{1+\left(\log t_{2}\right)^{\alpha-1}}\right|<\varepsilon, \quad\left|\frac{\left(\log t_{1}\right)^{\alpha-1}}{1+\left(\log t_{1}\right)^{\alpha-1}}-\frac{\left(\log t_{2}\right)^{\alpha-1}}{1+\left(\log t_{2}\right)^{\alpha-1}}\right|<\Gamma(\alpha) \varepsilon, \\
& \left|\frac{T_{1, \alpha}\left(t_{1}\right)}{1+\left(\log t_{1}\right)^{\alpha-1}}-\frac{T_{1, \alpha}\left(t_{2}\right)}{1+\left(\log t_{2}\right)^{\alpha-1}}\right|<\varepsilon, \quad\left|\frac{T_{2, \alpha}\left(t_{1}\right)}{1+\left(\log t_{1}\right)^{\alpha-1}}-\frac{T_{2, \alpha}\left(t_{2}\right)}{1+\left(\log t_{2}\right)^{\alpha-1}}\right|<\varepsilon .
\end{aligned}
$$

For $\forall u \in B, t_{1}, t_{2} \in J_{1}$, with $t_{1}<t_{2},\left|t_{1}-t_{2}\right|<\delta_{1}$,

$$
\begin{aligned}
& \left|\frac{A u\left(t_{1}\right)}{1+\left(\log t_{1}\right)^{\alpha-1}}-\frac{A u\left(t_{2}\right)}{1+\left(\log t_{2}\right)^{\alpha-1}}\right| \\
& \quad=\mid \int_{1}^{+\infty}\left(\frac{G_{\alpha}\left(t_{1}, s\right)}{1+\left(\log t_{1}\right)^{\alpha-1}}-\frac{G_{\alpha}\left(t_{2}, s\right)}{1+\left(\log t_{2}\right)^{\alpha-1}}\right) q(s) F_{u}(s) \frac{d s}{s}
\end{aligned}
$$




$$
\begin{aligned}
& \quad+\int_{1}^{+\infty}\left(\frac{T_{1, \alpha}\left(t_{1}\right)}{1+\left(\log t_{1}\right)^{\alpha-1}}-\frac{T_{1, \alpha}\left(t_{2}\right)}{1+\left(\log t_{2}\right)^{\alpha-1}}\right)\left(\int_{1}^{+\infty} g_{1}(t) G_{\alpha}(t, s) \frac{d t}{t}\right) q(s) F_{u}(s) \frac{d s}{s} \\
& +\int_{1}^{+\infty}\left(\frac{T_{2, \alpha}\left(t_{1}\right)}{1+\left(\log t_{1}\right)^{\alpha-1}}-\frac{T_{2, \alpha}\left(t_{2}\right)}{1+\left(\log t_{2}\right)^{\alpha-1}}\right)\left(\int_{1}^{+\infty} g_{2}(t) G_{\alpha}(t, s) \frac{d t}{t}\right) q(s) F_{u}(s) \frac{d s}{s} \mid \\
& \leq \int_{1}^{t_{2}}\left|\frac{G_{\alpha}\left(t_{1}, s\right)}{1+\left(\log t_{1}\right)^{\alpha-1}}-\frac{G_{\alpha}\left(t_{2}, s\right)}{1+\left(\log t_{2}\right)^{\alpha-1}}\right| q(s) f_{r_{1}}(s) \frac{d s}{s} \\
& +\frac{1}{\Gamma(\alpha)} \int_{t_{2}}^{+\infty}\left|\frac{\left(\log t_{1}\right)^{\alpha-1}}{1+\left(\log t_{1}\right)^{\alpha-1}}-\frac{\left(\log t_{2}\right)^{\alpha-1}}{1+\left(\log t_{2}\right)^{\alpha-1}}\right| q(s) f_{r_{1}}(s) \frac{d s}{s} \\
& +\varepsilon l_{1} \int_{1}^{+\infty} q(s) f_{r_{1}}(s) \frac{d s}{s}+\varepsilon \delta_{2} \int_{1}^{+\infty} q(s) f_{r_{1}}(s) \frac{d s}{s} \\
& \leq \varepsilon\left(1+l_{1}+\delta_{2}\right) \int_{1}^{+\infty} q(s) f_{r_{1}}(s) \frac{d s}{s} .
\end{aligned}
$$

Similarly, $\frac{G_{\alpha-\beta_{1}}(t, s)}{1+(\log t)^{\alpha-\beta_{1}-1}}$ is continuous on $J_{1} \times J_{1}$, and $\frac{(\log t)^{\alpha-\beta_{1}-1}}{1+(\log t)^{\alpha-\beta_{1}-1}}, \frac{T_{1, \alpha-\beta_{1}}(t)}{1+(\log t)^{\alpha-\beta_{1}-1}}, \frac{T_{2, \alpha-\beta_{1}}(t)}{1+(\log t)^{\alpha-\beta_{1}-1}}$ are continuous on $J_{1}$. For above $\varepsilon>0$, there is a constant $\delta_{2}>0$ such that, for all $t_{1}, t_{2} \in J_{1}$ with $t_{1}<t_{2},\left|t_{1}-t_{2}\right|<\delta_{2}$,

$$
\left|\frac{{ }^{H} D^{\beta_{1}} A u\left(t_{1}\right)}{1+\left(\log t_{1}\right)^{\alpha-\beta_{1}-1}}-\frac{{ }^{H} D^{\beta_{1}} A u\left(t_{2}\right)}{1+\left(\log t_{2}\right)^{\alpha-\beta_{1}-1}}\right| \leq \varepsilon\left(1+l_{1}+\delta_{2}\right) \int_{1}^{+\infty} q(s) f_{r_{1}}(s) \frac{d s}{s} .
$$

When $\beta_{2}<\alpha-1, \frac{\left(\log t t^{2+\beta_{2}-\alpha}\right.}{1+(\log t)^{\alpha-\beta_{2}}} G_{\alpha-\beta_{2}}(t, s)$ is continuous on $J_{1} \times J_{1}$, and $\frac{\log t}{1+(\log t)^{\alpha-\beta_{2}}}$, $\frac{(\log t)^{2+\beta_{2}-\alpha}}{1+(\log t)^{\alpha-\beta_{2}}} T_{1, \alpha-\beta_{2}}(t), \frac{(\log t)^{2+\beta_{2}-\alpha}}{1+(\log t)^{\alpha-\beta_{2}}} T_{2, \alpha-\beta_{2}}(t)$ are continuous on $J_{1}$. For above $\varepsilon>0$, there is a constant $\delta_{3}>0$ such that, for all $t_{1}, t_{2} \in J_{1}$ with $t_{1}<t_{2},\left|t_{1}-t_{2}\right|<\delta_{3}$,

$$
\begin{aligned}
& \left|\frac{\left(\log t_{1}\right)^{2+\beta_{2}-\alpha}}{1+\left(\log t_{1}\right)^{\alpha-\beta_{2}}}\left({ }^{H} D^{\beta_{2}} A u\right)\left(t_{1}\right)-\frac{\left(\log t_{2}\right)^{2+\beta_{2}-\alpha}}{1+\left(\log t_{2}\right)^{\alpha-\beta_{2}}}\left({ }^{H} D^{\beta_{2}} A u\right)\left(t_{2}\right)\right| \\
& \leq \int_{1}^{t_{2}}\left|\frac{\left(\log t_{1}\right)^{2+\beta_{2}-\alpha}}{1+\left(\log t_{1}\right)^{\alpha-\beta_{2}}} G_{\alpha-\beta_{2}}\left(t_{1}, s\right)-\frac{\left(\log t_{2}\right)^{2+\beta_{2}-\alpha}}{1+\left(\log t_{2}\right)^{\alpha-\beta_{2}}} G_{\alpha-\beta_{2}}\left(t_{2}, s\right)\right| q(s) f_{r_{1}}(s) \frac{d s}{s} \\
& +\frac{1}{\Gamma\left(\alpha-\beta_{2}\right)} \int_{t_{2}}^{+\infty}\left|\frac{\log t_{1}}{1+\left(\log t_{1}\right)^{\alpha-\beta_{2}}}-\frac{\log t_{2}}{1+\left(\log t_{2}\right)^{\alpha-\beta_{2}}}\right| q(s) f_{r_{1}}(s) \frac{d s}{s} \\
& +\left|\frac{\left(\log t_{1}\right)^{2+\beta_{2}-\alpha}}{1+\left(\log t_{1}\right)^{\alpha-\beta_{2}}} T_{1, \alpha-\beta_{2}}\left(t_{1}\right)-\frac{\left(\log t_{2}\right)^{2+\beta_{2}-\alpha}}{1+\left(\log t_{2}\right)^{\alpha-\beta_{2}}} T_{1, \alpha-\beta_{2}}\left(t_{2}\right)\right| l_{1} \int_{1}^{+\infty} q(s) f_{r_{1}}(s) \frac{d s}{s} \\
& +\left|\frac{\left(\log t_{1}\right)^{2+\beta_{2}-\alpha}}{1+\left(\log t_{1}\right)^{\alpha-\beta_{2}}} T_{2, \alpha-\beta_{2}}\left(t_{1}\right)-\frac{\left(\log t_{2}\right)^{2+\beta_{2}-\alpha}}{1+\left(\log t_{2}\right)^{\alpha-\beta_{2}}} T_{2, \alpha-\beta_{2}}\left(t_{2}\right)\right| \delta_{2} \int_{1}^{+\infty} q(s) f_{r_{1}}(s) \frac{d s}{s} \\
& \leq \varepsilon\left(1+l_{1}+\delta_{2}\right) \int_{1}^{+\infty} q(s) f_{r_{1}}(s) \frac{d s}{s}, \quad\left(\beta_{2}<\alpha-1\right) .
\end{aligned}
$$

When $\beta_{2}=\alpha-1, \frac{\log t}{1+\log t} G(t, s)$ is continuous on $J_{1} \times J_{1}$ and $\frac{\log t}{1+\log t}$ is continuous on $J_{1}$, there exists $\delta_{4}>0$ such that, for all $t_{1}, t_{2} \in J_{1}$ with $t_{1}<t_{2},\left|t_{1}-t_{2}\right|<\delta_{4}$,

$$
\begin{gathered}
\left|\frac{\left(\log t_{1}\right)^{2+\beta_{2}-\alpha}}{1+\left(\log t_{1}\right)^{\alpha-\beta_{2}}}\left({ }^{H} D^{\beta_{2}} A u\right)\left(t_{1}\right)-\frac{\left(\log t_{2}\right)^{2+\beta_{2}-\alpha}}{1+\left(\log t_{2}\right)^{\alpha-\beta_{2}}}\left({ }^{H} D^{\beta_{2}} A u\right)\left(t_{2}\right)\right| \\
\leq \int_{1}^{+\infty}\left|\frac{\log t_{1}}{1+\log t_{1}} G\left(t_{1}, s\right)-\frac{\log t_{2}}{1+\log t_{2}} G\left(t_{2}, s\right)\right| q(s) f_{r_{1}}(s) \frac{d s}{s} \\
\quad+\left|\frac{\log t_{1}}{1+\log t_{1}}-\frac{\log t_{2}}{1+\log t_{2}}\right| \delta l_{1} l_{2} \int_{1}^{+\infty} q(s) f_{r_{1}}(s) \frac{d s}{s}
\end{gathered}
$$




$$
\begin{aligned}
& +\left|\frac{\log t_{1}}{1+\log t_{1}}-\frac{\log t_{2}}{1+\log t_{2}}\right| \delta\left(1-\delta_{1}\right) \delta_{2} \int_{1}^{+\infty} q(s) f_{r_{1}}(s) \frac{d s}{s} \\
\leq & \varepsilon\left(1+\delta l_{1} l_{2}+\delta\left(1-\delta_{1}\right) \delta_{2}\right) \int_{1}^{+\infty} q(s) f_{r_{1}}(s) \frac{d s}{s} .
\end{aligned}
$$

Hence, $\frac{A u(t)}{1+(\log t)^{\alpha-1}}, \frac{{ }^{H} D^{\beta_{1}} A u(t)}{1+(\log t)^{\alpha-\beta_{1}-1}}, \frac{(\log t)^{2+\beta_{2}-\alpha}}{1+(\log t)^{\alpha-\beta_{2}}}\left({ }^{H} D^{\beta_{2}} A u\right)(t)$ are equicontinuous on $J_{1}$. (3) Now we indicate that the second condition (2) of Lemma 2.7 holds. Since

$$
\lim _{t \rightarrow+\infty} \frac{T_{1, \alpha}(t)}{1+(\log t)^{\alpha-1}}=\frac{\delta l_{2}}{\Gamma(\alpha)}, \quad \lim _{t \rightarrow+\infty} \frac{T_{2, \alpha}(t)}{1+(\log t)^{\alpha-1}}=\frac{\delta\left(1-\delta_{1}\right)}{\Gamma(\alpha)}
$$

for any $\varepsilon>0$, there exists a constant $T_{1}>1$ such that $\forall t_{1}, t_{2}>T_{1}$,

$$
\left|\frac{T_{1, \alpha}\left(t_{1}\right)}{1+\left(\log t_{1}\right)^{\alpha-1}}-\frac{T_{1, \alpha}\left(t_{2}\right)}{1+\left(\log t_{2}\right)^{\alpha-1}}\right|<\varepsilon, \quad\left|\frac{T_{2, \alpha}\left(t_{1}\right)}{1+\left(\log t_{1}\right)^{\alpha-1}}-\frac{T_{2, \alpha}\left(t_{2}\right)}{1+\left(\log t_{2}\right)^{\alpha-1}}\right|<\varepsilon .
$$

On the other hand, for $\forall u \in B$, we have

$$
\int_{1}^{+\infty} q(s)\left|F_{u}(s)\right| \frac{d s}{s} \leq \int_{1}^{+\infty} q(s) f_{r_{1}}(s) \frac{d s}{s}<+\infty
$$

Hence, for given $\varepsilon>0$, there exists a constant $L>0$ such that $\int_{L}^{+\infty} q(s) f_{r_{1}}(s) \frac{d s}{s}<\varepsilon$. Similarly, due to

$$
\lim _{t \rightarrow+\infty} \frac{G_{\alpha}(t, s)}{1+(\log t)^{\alpha-1}}=0, \quad 1 \leq s \leq L,
$$

there exists a constant $T_{2}>L$ such that, for any $t_{1}, t_{2}>T_{2}, 1 \leq s \leq L$, we have

$$
\left|\frac{G_{\alpha}\left(t_{1}, s\right)}{1+\left(\log t_{1}\right)^{\alpha-1}}-\frac{G_{\alpha}\left(t_{2}, s\right)}{1+\left(\log t_{2}\right)^{\alpha-1}}\right|<\varepsilon .
$$

Let $\forall t_{1}, t_{2}>\max \left\{T_{1}, T_{2}\right\}$, by Lemma 2.6 ,

$$
\begin{aligned}
& \left|\frac{A u\left(t_{1}\right)}{1+\left(\log t_{1}\right)^{\alpha-1}}-\frac{A u\left(t_{2}\right)}{1+\left(\log t_{2}\right)^{\alpha-1}}\right| \\
& \leq \int_{1}^{L}\left|\frac{G_{\alpha}\left(t_{1}, s\right)}{1+\left(\log t_{1}\right)^{\alpha-1}}-\frac{G_{\alpha}\left(t_{2}, s\right)}{1+\left(\log t_{2}\right)^{\alpha-1}}\right| q(s)\left|F_{u}(s)\right| \frac{d s}{s} \\
& +\int_{L}^{+\infty}\left|\frac{G_{\alpha}\left(t_{1}, s\right)}{1+\left(\log t_{1}\right)^{\alpha-1}}-\frac{G_{\alpha}\left(t_{2}, s\right)}{1+\left(\log t_{2}\right)^{\alpha-1}}\right| q(s)\left|F_{u}(s)\right| \frac{d s}{s} \\
& +\int_{1}^{+\infty}\left|\frac{T_{1, \alpha}\left(t_{1}\right)}{1+\left(\log t_{1}\right)^{\alpha-1}}-\frac{T_{1, \alpha}\left(t_{2}\right)}{1+\left(\log t_{2}\right)^{\alpha-1}}\right|\left(\int_{1}^{+\infty} g_{1}(t) G_{\alpha}(t, s) \frac{d t}{t}\right) q(s)\left|F_{u}(s)\right| \frac{d s}{s} \\
& +\int_{1}^{+\infty}\left|\frac{T_{2, \alpha}\left(t_{1}\right)}{1+\left(\log t_{1}\right)^{\alpha-1}}-\frac{T_{2, \alpha}\left(t_{2}\right)}{1+\left(\log t_{2}\right)^{\alpha-1}}\right|\left(\int_{1}^{+\infty} g_{2}(t) G_{\alpha}(t, s) \frac{d t}{t}\right) q(s)\left|F_{u}(s)\right| \frac{d s}{s} \\
& \leq \varepsilon \int_{1}^{L} q(s) f_{r_{1}}(s) \frac{d s}{s}+\frac{2}{\Gamma(\alpha)} \varepsilon+\varepsilon l_{1} \int_{1}^{+\infty} q(s) f_{r_{1}}(s) \frac{d s}{s}+\varepsilon \delta_{2} \int_{1}^{+\infty} q(s) f_{r_{1}}(s) \frac{d s}{s} \\
& \leq \varepsilon\left(1+l_{1}+\delta_{2}\right) \int_{1}^{+\infty} q(s) f_{r_{1}}(s) \frac{d s}{s}+\frac{2}{\Gamma(\alpha)} \varepsilon
\end{aligned}
$$


Similarly,

$$
\lim _{t \rightarrow+\infty} \frac{T_{1, \alpha-\beta_{1}}(t)}{1+(\log t)^{\alpha-\beta_{1}-1}}=\frac{\delta l_{2}}{\Gamma\left(\alpha-\beta_{1}\right)}, \quad \lim _{t \rightarrow+\infty} \frac{T_{2, \alpha-\beta_{1}}(t)}{1+(\log t)^{\alpha-\beta_{1}-1}}=\frac{\delta\left(1-\delta_{1}\right)}{\Gamma\left(\alpha-\beta_{1}\right)},
$$

there exists a constant $T_{3}>1$ such that $\forall t_{1}, t_{2}>T_{3}$,

$$
\begin{aligned}
& \left|\frac{T_{1, \alpha-\beta_{1}}\left(t_{1}\right)}{1+\left(\log t_{1}\right)^{\alpha-\beta_{1}-1}}-\frac{T_{1, \alpha-\beta_{1}}\left(t_{2}\right)}{1+\left(\log t_{2}\right)^{\alpha-\beta_{1}-1}}\right|<\varepsilon, \\
& \left|\frac{T_{2, \alpha-\beta_{1}}\left(t_{1}\right)}{1+\left(\log t_{1}\right)^{\alpha-\beta_{1}-1}}-\frac{T_{2, \alpha-\beta_{1}}\left(t_{2}\right)}{1+\left(\log t_{2}\right)^{\alpha-\beta_{1}-1}}\right|<\varepsilon .
\end{aligned}
$$

From $\lim _{t \rightarrow+\infty} \frac{G_{\alpha-\beta_{1}}(t, s)}{1+(\log t)^{\alpha-\beta_{1}-1}}=0,1 \leq s \leq L$, there exists a constant $T_{4}>L$ such that, for any $t_{1}, t_{2}>T_{4}, 1 \leq s \leq L$, we have $\left|\frac{G_{\alpha-\beta_{1}}\left(t_{1}, s\right)}{1+\left(\log t_{1}\right)^{\alpha-\beta} \beta_{1-1}}-\frac{G_{\alpha-\beta_{1}}\left(t_{2}, s\right)}{1+\left(\log t_{2}\right)^{\alpha-\beta} \beta_{1-1}}\right|<\varepsilon$. Let $\forall t_{1}, t_{2}>\max \left\{T_{3}, T_{4}\right\}$,

$$
\left|\frac{{ }^{H} D^{\beta_{1}} A u\left(t_{1}\right)}{1+\left(\log t_{1}\right)^{\alpha-\beta_{1}-1}}-\frac{{ }^{H} D^{\beta_{1}} A u\left(t_{2}\right)}{1+\left(\log t_{2}\right)^{\alpha-\beta_{1}-1}}\right| \leq \varepsilon\left(1+l_{1}+\delta_{2}\right) \int_{1}^{+\infty} q(s) f_{r_{1}}(s) \frac{d s}{s}+\frac{2}{\Gamma\left(\alpha-\beta_{1}\right)} \varepsilon .
$$

When $\beta_{2}<\alpha-1$,

$$
\begin{aligned}
& \lim _{t \rightarrow+\infty} \frac{(\log t)^{2+\beta_{2}-\alpha}}{1+(\log t)^{\alpha-\beta_{2}}} T_{1, \alpha-\beta_{2}}(t)=0, \\
& \lim _{t \rightarrow+\infty} \frac{(\log t)^{2+\beta_{2}-\alpha}}{1+(\log t)^{\alpha-\beta_{2}}} T_{2, \alpha-\beta_{2}}(t)=0,
\end{aligned}
$$

there exists a constant $T_{5}>1$ such that $\forall t_{1}, t_{2}>T_{5}$,

$$
\begin{aligned}
& \left|\frac{\left(\log t_{1}\right)^{2+\beta_{2}-\alpha}}{1+\left(\log t_{1}\right)^{\alpha-\beta_{2}}} T_{1, \alpha-\beta_{2}}\left(t_{1}\right)-\frac{\left(\log t_{2}\right)^{2+\beta_{2}-\alpha}}{1+\left(\log t_{2}\right)^{\alpha-\beta_{2}}} T_{1, \alpha-\beta_{2}}\left(t_{2}\right)\right|<\varepsilon, \\
& \left|\frac{\left(\log t_{1}\right)^{2+\beta_{2}-\alpha}}{1+\left(\log t_{1}\right)^{\alpha-\beta_{2}}} T_{2, \alpha-\beta_{2}}\left(t_{1}\right)-\frac{\left(\log t_{2}\right)^{2+\beta_{2}-\alpha}}{1+\left(\log t_{2}\right)^{\alpha-\beta_{2}}} T_{2, \alpha-\beta_{2}}\left(t_{2}\right)\right|<\varepsilon .
\end{aligned}
$$

From $\lim _{t \rightarrow+\infty} \frac{(\log t)^{2+\beta_{2}-\alpha}}{1+(\log t)^{\alpha-\beta_{2}}} G_{\alpha-\beta_{2}}(t, s)=0,1 \leq s \leq L$, there exists a constant $T_{6}>L$ such that, for any $t_{1}, t_{2}>T_{6}, 1 \leq s \leq L$, we have

$$
\left|\frac{\left(\log t_{1}\right)^{2+\beta_{2}-\alpha}}{1+\left(\log t_{1}\right)^{\alpha-\beta_{2}}} G_{\alpha-\beta_{2}}\left(t_{1}, s\right)-\frac{\left(\log t_{2}\right)^{2+\beta_{2}-\alpha}}{1+\left(\log t_{2}\right)^{\alpha-\beta_{2}}} G_{\alpha-\beta_{2}}\left(t_{1}, s\right)\right|<\varepsilon .
$$

Let $\forall t_{1}, t_{2}>\max \left\{T_{5}, T_{6}\right\}$,

$$
\begin{aligned}
& \left|\frac{\left(\log t_{1}\right)^{2+\beta_{2}-\alpha}}{1+\left(\log t_{1}\right)^{\alpha-\beta_{2}}}\left({ }^{H} D^{\beta_{2}} A u\right)\left(t_{1}\right)-\frac{\left(\log t_{2}\right)^{2+\beta_{2}-\alpha}}{1+\left(\log t_{2}\right)^{\alpha-\beta_{2}}}\left({ }^{H} D^{\beta_{2}} A u\right)\left(t_{2}\right)\right| \\
& \quad \leq \varepsilon\left(1+l_{1}+\delta_{2}\right) \int_{1}^{+\infty} q(s) f_{r_{1}}(s) \frac{d s}{s}+\frac{2}{\Gamma\left(\alpha-\beta_{2}\right)} \varepsilon .
\end{aligned}
$$

When $\beta_{2}=\alpha-1, \lim _{t \rightarrow+\infty} \frac{\log t}{1+\log t}=1$, there exists a constant $T_{7}>1$ such that $\forall t_{1}, t_{2}>T_{7}$,

$$
\left|\frac{\log t_{1}}{1+\log t_{1}}-\frac{\log t_{2}}{1+\log t_{2}}\right|<\varepsilon
$$


$\lim _{t \rightarrow+\infty} \frac{\log t}{1+\log t} G(t, s)=0,1 \leq s \leq L$, there exists a constant $T_{8}>L$ such that, for any $t_{1}, t_{2}>$ $T_{8}, 1 \leq s \leq L$, we have

$$
\left|\frac{\log t_{1}}{1+\log t_{1}} G\left(t_{1}, s\right)-\frac{\log t_{2}}{1+\log t_{2}} G\left(t_{2}, s\right)\right|<\varepsilon
$$

Let $\forall t_{1}, t_{2}>\max \left\{T_{7}, T_{8}\right\}$,

$$
\begin{aligned}
& \left|\frac{\left(\log t_{1}\right)^{2+\beta_{2}-\alpha}}{1+\left(\log t_{1}\right)^{\alpha-\beta_{2}}}\left({ }^{H} D^{\beta_{2}} A u\right)\left(t_{1}\right)-\frac{\left(\log t_{2}\right)^{2+\beta_{2}-\alpha}}{1+\left(\log t_{2}\right)^{\alpha-\beta_{2}}}\left({ }^{H} D^{\beta_{2}} A u\right)\left(t_{2}\right)\right| \\
& \quad \leq \varepsilon\left(1+\delta l_{1} l_{2}+\delta\left(1-\delta_{1}\right) \delta_{2}\right) \int_{1}^{+\infty} q(s) f_{r_{1}}(s) \frac{d s}{s}+2 \varepsilon .
\end{aligned}
$$

Combining (2) and (3), from Lemma 2.7, we have $A(B)$ is relatively compact. Therefore we conclude that $A$ is a compact operator. Hence, $A$ is completely continuous in $Y$.

Step 2. $A\left(\Omega_{R}\right) \subset \Omega_{R}$, where $\Omega_{R}=\{u \in Y:\|u\| \leq R\}$.

For any $u \in \Omega_{R}$, we know that

$$
\frac{|u(t)|}{1+(\log t)^{\alpha-1}} \leq R, \quad \frac{\left|{ }^{H} D^{\beta_{1}} u(t)\right|}{1+(\log t)^{\alpha-\beta_{1}-1}} \leq R,\left.\quad \frac{(\log t)^{2+\beta_{2}-\alpha}}{1+(\log t)^{\alpha-\beta_{2}}}\right|^{H} D^{\beta_{2}} u(t) \mid \leq R .
$$

From condition $\left(H_{3}\right)$ and (3.5)-(3.8), we have

$$
\begin{aligned}
& \left|\frac{A u(t)}{1+(\log t)^{\alpha-1}}\right|=\left|\int_{1}^{+\infty} \frac{K_{\alpha}(t, s)}{1+(\log t)^{\alpha-1}} q(s) F_{u}(s) \frac{d s}{s}\right| \\
& \leq \Delta_{1} \int_{1}^{+\infty} q(s) f_{R}(s) \frac{d s}{s} \leq R . \\
& \left|\frac{{ }^{H} D^{\beta_{1}} A u(t)}{1+(\log t)^{\alpha-\beta_{1}-1}}\right|=\left|\int_{1}^{+\infty} \frac{K_{\alpha-\beta_{1}}(t, s)}{1+(\log t)^{\alpha-\beta_{1}-1}} q(s) F_{u}(s) \frac{d s}{s}\right| \\
& \leq \Delta_{2} \int_{1}^{+\infty} q(s) f_{R}(s) \frac{d s}{s} \leq R \\
& \frac{(\log t)^{2+\beta_{2}-\alpha}}{1+(\log t)^{\alpha-\beta_{2}}}\left|{ }^{H} D^{\beta_{2}} A u(t)\right|=\left|\int_{1}^{+\infty} \frac{(\log t)^{2+\beta_{2}-\alpha}}{1+(\log t)^{\alpha-\beta_{2}}} K_{\alpha-\beta_{2}}(t, s) q(s) F_{u}(s) \frac{d s}{s}\right| \\
& \leq \Delta_{3} \int_{1}^{+\infty} q(s) f_{R}(s) \frac{d s}{s} \leq R, \quad\left(\beta_{2}<\alpha-1\right) \\
& \frac{(\log t)^{2+\beta_{2}-\alpha}}{1+(\log t)^{\alpha-\beta_{2}}}\left|{ }^{H} D^{\beta_{2}} A u(t)\right|=\left|\int_{1}^{+\infty} \frac{(\log t)^{2+\beta_{2}-\alpha}}{1+(\log t)^{\alpha-\beta_{2}}} K^{\beta_{2}}(t, s) q(s) F_{u}(s) \frac{d s}{s}\right| \\
& \leq \Delta_{4} \int_{1}^{+\infty} q(s) f_{R}(s) \frac{d s}{s} \leq R, \quad\left(\beta_{2}=\alpha-1\right),
\end{aligned}
$$

which means that $\|A u\|_{Y} \leq R, A\left(\Omega_{R}\right) \subset \Omega_{R}$ holds.

Step 3. We will show that $A$ has at least one solution in $Y$.

By step 1 and step 2, we have $A: \Omega_{R} \rightarrow \Omega_{R}$ is completely continuous. $\Omega_{R}$ is a nonempty, closed, bounded, and convex subset of $Y$. According to the Schauder fixed point theorem, we conclude that $A$ has at least one fixed point in $\Omega_{R}$, then boundary value problem (1.1), (1.2) has at least one solution. 


$$
\text { Let } \begin{aligned}
J_{0} & =(1,+\infty), L(s)=\max \left\{1+(\log s)^{\alpha-1}, 1+(\log s)^{\alpha-\beta_{1}-1}, \frac{1+(\log s)^{\alpha-\beta_{2}}}{(\log s)^{2+\beta_{2}-\alpha}}\right\}, s \in J_{0} . \\
Y_{1} & =\left\{y \in L\left(J_{0}, \mathbb{R}^{+}\right): \int_{1}^{+\infty} y(s) L(s) q(s) \frac{d s}{s}<+\infty\right\} .
\end{aligned}
$$

Corollary 3.1 Assume that conditions $\left(\mathbf{H}_{2}\right)-\left(\mathbf{H}_{3}\right)$ are satisfied. Further assume that the following condition $\left(\mathbf{H}_{4}\right)$ holds:

$\left(\mathbf{H}_{4}\right) f(t, u, v, w)$ is continuous on $J \times \mathbb{R}^{3}$, and there exist nonnegative functions $a, b, c, d \in$ $Y_{1}$ such that, for all $(u, v, w) \in \mathbb{R}^{3}$ and $t \in J$,

$$
|f(t, u, v, w)| \leq a(t)+b(t)|u|+c(t)|v|+d(t)|w|
$$

Then boundary value problem (1.1) and (1.2) has at least one solution.

Proof Let $\Omega_{R}=\left\{u \in Y:\|u\|_{Y} \leq R\right\}$. For any $u \in \Omega_{R}, s \in J$, we have

$$
\begin{aligned}
\left|F_{u}(s)\right|= & \left|f\left(s, u(s),{ }^{H} D^{\beta_{1}} u(s),{ }^{H} D^{\beta_{2}} u(s)\right)\right| \\
\leq & a(s)+b(s) u(s)+c(s)^{H} D^{\beta_{1}} u(s)+d(s)^{H} D^{\beta_{2}} u(s) \\
= & a(s)+b(s)\left(1+(\log s)^{\alpha-1}\right) \frac{u(s)}{1+(\log s)^{\alpha-1}} \\
& +c(s)\left(1+(\log s)^{\alpha-\beta-1}\right) \frac{{ }^{H} D^{\beta} u(s)}{1+(\log s)^{\alpha-\beta_{1}-1}} \\
& +d(s) \frac{1+(\log s)^{\alpha-\beta_{2}}}{(\log s)^{2+\beta_{2}-\alpha}} \frac{(\log s)^{2+\beta_{2}-\alpha}}{1+(\log s)^{\alpha-\beta_{2}}}\left({ }^{H} D^{\beta_{2}} u\right)(s) \\
\leq & a(s)+R\left[b(s)\left(1+(\log s)^{\alpha-1}\right)+c(s)\left(1+(\log s)^{\alpha-\beta_{1}-1}\right)+d(s) \frac{1+(\log s)^{\alpha-\beta_{2}}}{(\log s)^{2+\beta_{2}-\alpha}}\right] \\
\triangleq & f_{R}(s) .
\end{aligned}
$$

Obviously,

$$
\begin{aligned}
& \int_{1}^{+\infty} a(s) q(s) \frac{d s}{s} \leq \int_{1}^{+\infty} a(s) L(s) q(s) \frac{d s}{s}<+\infty \\
& \int_{1}^{+\infty} b(s)\left(1+(\log s)^{\alpha-1}\right) q(s) \frac{d s}{s} \leq \int_{1}^{+\infty} b(s) L(s) q(s) \frac{d s}{s}<+\infty \\
& \int_{1}^{+\infty} c(s)\left(1+(\log s)^{\alpha-\beta_{1}-1}\right) q(s) \frac{d s}{s} \leq \int_{1}^{+\infty} c(s) L(s) q(s) \frac{d s}{s}<+\infty \\
& \int_{1}^{+\infty} d(s) \frac{1+(\log s)^{\alpha-\beta_{2}}}{(\log s)^{2+\beta_{2}-\alpha}} q(s) \frac{d s}{s} \leq \int_{1}^{+\infty} d(s) L(s) q(s) \frac{d s}{s}<+\infty
\end{aligned}
$$

hence $\int_{1}^{+\infty} q(s) f_{R}(s) \frac{d s}{s}<+\infty$.

By Theorem 3.1, we get that problem (1.1), (1.2) has at least one solution. 


\section{Example}

Example 4.1 Consider the Hadamard-type fractional boundary value problem on unbounded domain:

$$
\left\{\begin{array}{l}
{ }^{H} D^{\frac{5}{2}} u(t)+e^{-t} \frac{\left.\left.t^{2}(\log t)^{\frac{3}{4}} u(t)\right)^{H} D^{\frac{1}{4}} u(t)\right)\left(H^{H} D^{\frac{5}{4}} u(t)\right)}{\left(1+(\log t)^{\frac{3}{2}}\right)\left(\left(1+(\log t)^{\frac{5}{4}}\right)\left(1+(\log t)^{\frac{5}{4}}\right)\right.}=0, \quad 1<t<+\infty, \\
u(1)=0, \\
{ }^{H} D^{\frac{1}{2}} u(1)=\int_{1}^{+\infty} \frac{u(s)}{s^{3}(\log s)^{\frac{1}{2}} \frac{d s}{s}} \\
{ }^{H} D^{\frac{3}{2}} u(+\infty)=\int_{1}^{+\infty} \frac{u(s)}{s^{3}(\log s)^{\frac{1}{2}}} \frac{d s}{s} .
\end{array}\right.
$$

Let $\alpha=\frac{5}{2}, \beta_{1}=\frac{1}{4}, \beta_{2}=\frac{5}{4}, q(t)=e^{-t}, g_{1}(t)=g_{2}(t)=\frac{1}{t^{3}(\log t)^{\frac{1}{2}}}, f_{r}(t)=t^{2} r^{3}$,

$$
f(t, u, v, w)=\frac{t^{2}(\log t)^{\frac{3}{4}} u v w}{\left(1+(\log t)^{\frac{3}{2}}\right)\left(1+(\log t)^{\frac{5}{4}}\right)\left(1+(\log t)^{\frac{5}{4}}\right)} .
$$

Hence

$$
\begin{aligned}
& f\left(t,\left(1+(\log t)^{\frac{3}{2}}\right) u,\left(1+(\log t)^{\frac{5}{4}}\right) v, \frac{1+(\log t)^{\frac{5}{4}}}{(\log t)^{\frac{3}{4}}} w\right)=t^{2} u v w \leq t^{2} r^{3}=f_{r}(t), \\
& \forall t \in J_{1}, 0 \leq u, v, w \leq r . \\
& \int_{1}^{+\infty} f_{r}(t) q(t) \frac{d t}{t}=r^{3} \int_{1}^{+\infty} t^{2} e^{-t} d t=5 e^{-1} r^{3} . \\
& l_{1}=\delta_{2}=\frac{1}{\Gamma\left(\frac{5}{2}\right)} \int_{1}^{+\infty} \frac{1}{t^{3}(\log t)^{\frac{1}{2}}}(\log t)^{\frac{3}{2}} \frac{d t}{t}=\frac{1}{9 \Gamma\left(\frac{5}{2}\right)} \approx 0.0836<1, \\
& l_{2}=\delta_{1}=\frac{1}{\Gamma\left(\frac{3}{2}\right)} \int_{1}^{+\infty} \frac{1}{t^{3}(\log t)^{\frac{1}{2}}}(\log t)^{\frac{1}{2}} \frac{d t}{t}=\frac{1}{3 \Gamma\left(\frac{3}{2}\right)} \approx 0.3761<1, \\
& \text { and } \frac{1}{\delta}=\left(1-\delta_{1}\right)\left(1-\delta_{2}\right)-l_{1} l_{2} \approx 0.5403>0 \text {. } \\
& \Delta_{1}=\frac{1}{\Gamma(\alpha)}+l_{1}\left(\frac{\delta l_{2}}{\Gamma(\alpha)}+\frac{\delta\left(1-\delta_{2}\right)}{\Gamma(\alpha-1)}\right)+\delta_{2}\left(\frac{\delta\left(1-\delta_{1}\right)}{\Gamma(\alpha)}+\frac{\delta l_{1}}{\Gamma(\alpha-1)}\right) \\
& =\frac{1}{3 \Gamma\left(\frac{3}{2}\right)}\left(2+5 l_{1} \delta\right) \approx 1.0432 \text {, } \\
& \Delta_{2}=\frac{1}{\Gamma\left(\alpha-\beta_{1}\right)}+l_{1}\left(\frac{\delta l_{2}}{\Gamma\left(\alpha-\beta_{1}\right)}+\frac{\delta\left(1-\delta_{2}\right)}{\Gamma\left(\alpha-\beta_{1}-1\right)}\right) \\
& +\delta_{2}\left(\frac{\delta\left(1-\delta_{1}\right)}{\Gamma\left(\alpha-\beta_{1}\right)}+\frac{\delta l_{1}}{\Gamma\left(\alpha-\beta_{1}-1\right)}\right) \\
& =\frac{1}{5 \Gamma\left(\frac{5}{4}\right)}\left(4+9 l_{1} \delta\right) \approx 1.1899 \text {, }
\end{aligned}
$$




$$
\begin{aligned}
\Delta_{3}= & \frac{1}{\Gamma\left(\alpha-\beta_{2}\right)}+l_{1}\left(\frac{\delta l_{2}}{\Gamma\left(\alpha-\beta_{2}\right)}+\frac{\delta\left(1-\delta_{2}\right)}{\Gamma\left(\alpha-\beta_{2}-1\right)}\right) \\
& +\delta_{2}\left(\frac{\delta\left(1-\delta_{1}\right)}{\Gamma\left(\alpha-\beta_{2}\right)}+\frac{\delta l_{1}}{\Gamma\left(\alpha-\beta_{2}-1\right)}\right) \\
= & \frac{4}{5 \Gamma\left(\frac{1}{4}\right)}\left(1+\frac{5}{4} l_{1} \delta\right) \approx 1.3166, \\
\Delta_{4}= & 1+\delta l_{1} l_{2}+\delta\left(1-\delta_{1}\right) \delta_{2}=1+\delta l_{1} \approx 1.1547 .
\end{aligned}
$$

Therefore, conditions $\left(H_{1}\right)\left(H_{2}\right)$ hold. Choose $\Delta=\max \left\{\Delta_{1}, \Delta_{2}, \Delta_{3}, \Delta_{4}\right\}=\Delta_{3}, R \leq \sqrt{\frac{e}{5 \Delta}}$, then $\Delta \int_{1}^{+\infty} f_{R}(t) e^{-t} \frac{d t}{t}=5 \Delta e^{-1} R^{3} \leq R$, so condition $\left(H_{3}\right)$ holds. By Theorem 3.1, BVP (4.1) has at least one solution.

\section{Acknowledgements}

The authors would like to thank the referees for their useful suggestions which have significantly improved the paper

\section{Funding}

This research was supported by the National Natural Science Foundation of China (11871302).

\section{Availability of data and materials}

Not applicable.

\section{Competing interests}

The author declares that there is no conflict of interests regarding the publication of this paper.

\section{Authors' contributions}

All authors contributed equally and significantly in writing this article. All authors read and approved the final manuscript.

\section{Author details}

'School of Mathematics Sciences, Qufu Normal University, Qufu, People's Republic of China. ${ }^{2}$ Department of Mathematics and Statistics, Curtin University, Perth, Australia.

\section{Publisher's Note}

Springer Nature remains neutral with regard to jurisdictional claims in published maps and institutional affiliations.

Received: 21 April 2020 Accepted: 26 May 2020 Published online: 09 June 2020

\section{References}

1. Kilbas, A., Srivastava, H., Trujillo, J.: Theory and Applications of Fractional Differential Equations, vol. 204 Elsevier, Amsterdam (2006)

2. Hao, X.A., Sun, H., Liu, L.S.: Existence results for fractional integral boundary value problem involving fractional derivatives on an infinite interval. Math. Methods Appl. Sci. 41, 6984-6996 (2018)

3. Wang, F., Liu, L.S., Kong, D.B., Wu, Y.H.: Existence and uniqueness of positive solutions for a class of nonlinear fractional differential equations with mixed-type boundary value conditions. Nonlinear Anal., Model. Control 24(1), 73-94 (2019)

4. He, J.X., Zhang, X.G., Liu, L.S., Wu, Y.H., Cui, Y.J.: A singular fractional Kelvin-Voigt model involving a nonlinear operator and their convergence properties. Bound. Value Probl. 2017, 112 (2019)

5. Guo, L.M., Liu, L.S.: Unique iterative positive solutions for singular p-Laplacian fractional differential equation system with infinite-point boundary conditions. Bound. Value Probl. 2019, 113 (2019)

6. Wang, F., Liu, L.S., Wu, Y.H.: Iterative unique positive solutions for a new class of nonlinear singular higher order fractional differential equations with mixed-type boundary value conditions. J. Inequal. Appl. 2019, 210 (2019)

7. Zhu, B., Liu, L.S., Wu, Y.H.: Existence and uniqueness of global mild solutions for a class of nonlinear fractional reaction-diffusion equations with delay. Comput. Math. Appl. 78(6), 1811-1818 (2019)

8. Wang, F., Liu, L.S., Wu, Y.H., Zou, Y.M.: Iterative analysis of the unique positive solution for a class of singular nonlinear boundary value problems involving two types of fractional derivative with p-Laplacian operator. Complexity 2019, Article ID 2319062 (2019)

9. Min, D.D., Liu, L.S., Wu, Y.H.: Uniqueness of positive solution for the singular fractional differential equations involving integral boundary value conditions. Bound. Value Probl. 2018, 23 (2018)

10. Liu, X.Q., Liu, L.S., Wu, Y.H.: Existence of positive solutions for a singular nonlinear fractional differential equation with integral boundary conditions involving fractional derivatives. Bound. Value Probl. 2018, 24 (2018)

11. Guo, L.M., Liu, L.S., Wu, Y.H.: Iterative unique positive solutions for singular p-Laplacian fractional differential equation system with several parameters. Nonlinear Anal., Model. Control 23(2), 182-203 (2018)

12. Wu, J., Zhang, X.G., Liu, L.S., Wu, Y.H., Cui, Y.J.: The convergence analysis and error estimation for unique solution of a p-Laplacian fractional differential equation with singular decreasing nonlinearity. Bound. Value Probl. 2018, 82 (2018) 
13. Zhang, X.Q., Liu, L.S., Wu, Y.H., Zou, Y.M.: Existence and uniqueness of solutions for systems of fractional differential equations with Riemann-Stieltjes integral boundary condition. Adv. Differ. Equ. 2018, 204 (2018)

14. Wu, J., Zhang, X.G., Liu, L.S., Wu, Y.H., Cui, Y.J.: Convergence analysis of iterative scheme and error estimation of positive solution for a fractional differential equation. Math. Model. Anal. 23(4), 611-626 (2018)

15. Guo, L.M., Liu, L.S.: Maximal and minimal iterative positive solutions for singular infinite-point p-Laplacian fractional differential equations. Nonlinear Anal., Model. Control 23(6), 851-865 (2018)

16. He, J.X., Zhang, X.G., Liu, L.S., Cui, Y.J.: Existence and asymptotic behavior of solutions for a singular nonlocal fractional differential equation with nonlocal boundary conditions. Bound. Value Probl. 2018, 189 (2018)

17. Zhang, X.G., Liu, L.S., Wu, Y.H., Wiwatanapataphee, B.: Nontrivial solutions for a fractional advection dispersion equation in anomalous diffusion. Appl. Math. Lett. 66, 1-8 (2017)

18. Liu, L.S., Sun, F.L., Zhang, X.G., Wu, Y.H.: Bifurcation analysis for a singular differential system with two parameters via to degree theory. Nonlinear Anal., Model. Control 22(1), 31-50 (2017)

19. Liu, L.S., Li, H.D., Liu, C., Wu, Y.H.: Existence and uniqueness of positive solutions for singular fractional differential systems with coupled integral boundary value problems. J. Nonlinear Sci. Appl. 10, 243-262 (2017)

20. Wang, Y., Liu, L.S.: Uniqueness and existence of positive solutions for the fractional integro-differential equation. Bound. Value Probl. 2017, 12 (2017)

21. Zhang, X.G., Mao, C.L., Liu, L.S., Wu, Y.H.: Exact iterative solution for an abstract fractional dynamic system model for bioprocess. Qual. Theory Dyn. Syst. 16, 205-222 (2017)

22. Ren, T., Li, S.D., Zhang, X.G., Liu, L.S.: Maximum and minimum solutions for a nonlocal p-Laplacian fractional differential system from eco-economical processes. Bound. Value Probl. 2017, 118 (2017)

23. Zhu, B., Liu, L.S., Wu, Y.H.: Local and global existence of mild solutions for a class of semilinear fractional integro-differential equations. Fract. Calc. Appl. Anal. 20(6), 1338-1355 (2017)

24. Sun, F.L., Liu, L.S., Zhang, X.G., Wu, Y.H.: Spectral analysis for a singular differential system with integral boundary conditions. Mediterr. J. Math. 13, 4763-4782 (2016)

25. Liu, L.S., Zhang, X.Q., Jiang, J., Wu, Y.H.: The unique solution of a class of sum mixed monotone operator equations and its application to fractional boundary value problems. J. Nonlinear Sci. Appl. 9(5), 2943-2958 (2016)

26. Wu, J., Zhang, X.G., Liu, L.S., Wu, Y.H.: Twin iterative solutions for a fractional differential turbulent flow model. Bound. Value Probl. 2016, 98 (2016)

27. Wu, J., Zhang, X.G., Liu, L.S., Wu, Y.H., Wiwatanapataphee, B.: Iterative algorithm and estimation of solution for a fractional order differential equation. Bound. Value Probl. 2016, 116 (2016)

28. Zhu, B., Liu, L.S., Wu, Y.H.: Local and global existence of mild solutions for a class of nonlinear fractional reaction-diffusion equation with delay. Appl. Math. Lett. 61, 73-79 (2016)

29. Guo, L.M., Liu, L.S., Wu, Y.H.: Existence of positive solutions for singular higher-order fractional differential equations with infinite-points boundary conditions. Bound. Value Probl. 2016, 114 (2016)

30. Guo, L.M., Liu, L.S., Wu, Y.H.: Existence of positive solutions for singular fractional differential equations with infinite-point boundary conditions. Nonlinear Anal., Model. Control 21(5), 635-650 (2016)

31. Guo, L.M., Liu, L.S., Wu, Y.H.: Uniqueness of iterative positive solutions for the singular fractional differential equations with integral boundary conditions. Bound. Value Probl. 2016, 147 (2016)

32. Wang, F., Liu, L.S., Wu, Y.H.: A numerical algorithm for a class of fractional BVPs p-Laplacian operator and singularity —-the convergence and dependence analysis. Appl. Math. Comput. 382, 125339 (2020). https://doi.org/10.1016/j.amc.2020.125339.

33. Jiang, J.Q., O'Regan, D., Xu, J.F., Cui, Y.J.: Positive solutions for a class of p-Laplacian Hadamard fractional-order three-point boundary value problems. Mathematics 7, 439 (2019)

34. Zhang, H.Y., Li, Y.H., Xu, J.F.: Positive solutions for a system of fractional integral boundary value problems involving Hadamard-type fractional derivatives. Complexity 2019, Article ID 2671539 (2019)

35. Wang, G.T., Pei, K., Agarwal, R., Zhang, L., Ahmad, B.: Nonlocal Hadamard fractional boundary value problem with Hadamard integral and discrete boundary conditions on a half-line. J. Comput. Appl. Math. 342, 23-239 (2018)

36. Jiang, J.Q., O'Regan, D., Xu, J.F., Fu, Z.Q.: Positive solutions for a system of nonlinear Hadamard fractional differential equations involving coupled integral boundary conditions. J. Inequal. Appl. 2019, 204, 1-18 (2019)

37. Asawasamrit, S., Ntouyas, S., Tariboon, J., Nithiarayaphaks, W.: Coupled systems of sequential Caputo and Hadamard fractional differential equations with coupled separated boundary conditions. Symmetry 10(12), 701 (2018)

38. Yang, W.: Positive solutions for singular coupled integral boundary value problems of nonlinear Hadamard fractional differential equations. J. Nonlinear Sci. Appl. 8, 110-129 (2015)

39. Zhai, C.B., Wang, W., Li, H.: A uniqueness method to a new Hadamard fractional differential system with four-point boundary conditions. J. Inequal. Appl. 2018, 207 (2018)

40. Zhang, K., Fu, Z:: Solutions for a class of Hadamard fractional boundary value problems with sign-changing nonlinearity. J. Funct. Spaces 2019, Article ID 9046472 (2019)

41. Zhang, K., Wang, J., Ma, W.: Solutions for integral boundary value problems of nonlinear Hadamard fractional differential equations. J. Funct. Spaces 2018, Article ID 2193234 (2018)

42. Benhamida, W., Graef, J.R., Hamani, S.: Boundary value problems for Hadamard fractional differential equations with nonlocal multi-point boundary conditions. Fract. Differ. Calc. 8(1), 165-176 (2018)

43. Yukunthorn, W., Ahmad, B., Ntouyas, S.K., Tariboon, J.: On Caputo-Hadamard type fractional impulsive hybrid systems with nonlinear fractional integral conditions. Nonlinear Anal. Hybrid Syst. 19, 77-92 (2016)

44. Yukunthorn, W., Suantai, S., Ntouyas, S.K., Tariboon, J.: Boundary value problems for impulsive multi-order Hadamard fractional differential equations. Bound. Value Probl. 2015, 148 (2015)

45. Ardjouni, A.: Positive solutions for nonlinear Hadamard fractional differential equations with integral boundary conditions. AIMS Math. 4(4), 1101-1113 (2019)

46. Pei, K., Wang, G., Sun, Y.: Successive iterations and positive extremal solutions for a Hadamard type fractional integro-differential equations on infinite domain. Appl. Math. Comput. 312, 158-168 (2017)

47. El-Sayed, A.M.A., Gaafar, F.M.: Positive solutions of singular Hadamard-type fractional differential equations with infinite-point boundary conditions or integral boundary conditions. Adv. Differ. Equ. 2019, 382 (2019)

48. Zhang, W., Liu, W.: Existence of solutions for several higher-order Hadamard-type fractional differential equations with integral boundary conditions on infinite interval. Bound. Value Probl. 2018134 (2018) 
49. Thiramanus, P., Ntouyas, S., Tariboon, J.: Positive solutions for Hadamard fractional differential equations on infinite domain. Adv. Differ. Equ. 2016, 83 (2016)

50. Wang, G., Pei, K., Baleanu, D.: Explicit iteration to Hadamard fractional integro-differential equations on infinite domain. Adv. Differ. Equ. 2016, 299 (2016)

51. Rao, S.N., Singh, M., Meetei, M.Z.: Multiplicity of positive solutions for Hadamard fractional differential equations with p-Laplacian operator. Bound. Value Probl. 2020, 43 (2020)

Submit your manuscript to a SpringerOpen ${ }^{\circ}$ journal and benefit from:

- Convenient online submission

$\checkmark$ Rigorous peer review

Open access: articles freely available online

- High visibility within the field

- Retaining the copyright to your article

Submit your next manuscript at $\gg$ springeropen.com 